\title{
Hybrid Transceivers Design for Large-Scale Antenna Arrays Using Majorization-Minimization Algorithms
}

\author{
Aakash Arora Student Member, IEEE, Christos G. Tsinos Member, IEEE, \\ Bhavani Shankar Mysore R Member, IEEE, Symeon Chatzinotas Senior Member, IEEE, \\ Björn Ottersten Fellow, IEEE
}

\begin{abstract}
Hybrid analog-digital (A/D) transceivers are an appealing solution to reduce the transceiver hardware complexity and power consumption for the millimeter wave (mmWave) communication and more general large-scale antenna array (LSAA) systems. In contrast to fully digital conventional multipleinput-multiple-output (MIMO) systems, the baseband precoding operation splits into a lower-dimensional digital precoder followed by a network of analog phase shifters. In this paper, we consider the hybrid precoder design as a constant modulus constrained matrix factorization (CMCMF) problem for the most common types of hybrid architectures namely, the fully and the partially connected ones. Two lines of algorithms based on the majorization-minimization (MM) and the minorizationmaximization framework, respectively are proposed for these architectures. In particular, we present efficient algorithms scalable for LSAA systems with provable convergence guarantees to a stationary point. We also consider the hybrid postcoder design at the receiver end. Simulation results demonstrate that the proposed algorithms converge faster to a stationary point as compared to the state-of-the-art solutions that exist in literature. Furthermore, the solution tailored for the partial connected case achieves significantly improved performance in terms of the system spectral efficiency when compared to the existing solutions.
\end{abstract}

Index Terms-Hybrid precoding, majorization-minimization, MM, mmWave MIMO communications, alternating minimization, large-scale antenna arrays.

\section{INTRODUCTION}

Large-scale antenna arrays (LSAAs) are generally used in massive MIMO systems and are an essential requirement for a mmWave communication system. This is due to a fact that the mmWave frequencies suffer from several losses including propagation, penetration loss, etc., [1]-[5]. Thus, LSAAs are generally employed at the transceivers to achieve beamforming gains and combat the losses associated with the system. This is also feasible from the manufacturing perspective where many antenna elements are closely packed, due to shorter wavelengths at the mmWave frequencies. However, to build such a fully digital transceiver, in general, each antenna element

The authors are with the Interdisciplinary Centre for Security, Reliability and Trust (SnT), University of Luxembourg, Luxembourg. Part of this work was presented in IEEE GLOBECOM 2019.

Emails: \{aakash.arora,christos.tsinos, bhavani.shankar,symeon.chatzinotas, bjorn.ottersten\}@uni.lu.

This work is supported by the National Research Fund (FNR), Luxembourg under the AFR-PPP grant for Ph.D. project SPASAT (Ref.: 11607283), the CORE-PPP project PROSAT, ECLECTIC, and CI-PHY. has to be driven by a radio frequency (RF) chain. This strict requirement of a single RF chain per antenna substantially increases the hardware complexity and power consumption, when LSAAs are employed at the transceiver.

To address the aforementioned issues several precoding/beamforming approaches have been proposed in the literature. At first, analog-only beamforming was considered in [6]-[8], in which a bank of analog phase shifters is driven using a single RF chain and may support only single-stream transmission. But, this technique is not suitable for a MIMO system in which several streams are multiplexed. As an alternative, the phase-shifting network is replaced by analog switches [9]-[11], which substantially degrades the array gain.

In order to have a high beamforming gain while supporting simultaneous multiple streams transmission, hybrid A/D transceivers are proposed in [1]. In this architecture, the precoding operation splits into a lower-dimensional digital baseband precoder followed by a network of analog phase shifters. A similar architecture can also be considered on the receiver's side. The development of the hybrid precoder is based on the fact that the number of RF chains is limited by the number of transmit streams whereas the array gain is proportional to the number of antenna elements.

\section{A. Literature Review}

For mmWave systems, the hybrid transceivers were first introduced in [2], [3] and it was shown that the near-optimal hybrid precoders can be designed by maximizing the spectral efficiency (SE). In the literature, a variety of works exist for the hybrid precoder design [1]-[3], [5], [12]-[25]. These works can be classified into two categories namely, codebook based and codebook free approaches. In the codebook based design, the analog and digital precoders are precomputed and stored offline in a codebook structure [3], [13], [14]. This facilitates limited channel feedback from the user to the base station (BS). This procedure saves a significant amount of computation involved in computing the hybrid precoders [13]. In codebook free design, the problem of SE maximization is directly addressed [12] and the optimal precoders are designed with some approximations of the original problem. As already mentioned, there are many works available in the literature considering different problems associated with hybrid precoders design. First, we briefly summarize some of 
the recent works, then we proceed towards our problem of interest.

In [13], a hybrid beamforming design is proposed to determine the number of RF chains and phase shifters required to achieve the performance of a fully digital precoder. The problem with finite resolution phase shifters is considered in [14] and shown that the performance using 2-bit analog phase shifters is shown to be comparable to unquantized phase shifters. A hybrid design with a reduced number of phase shifters is proposed in [15]. Apart from SE maximization, the problem of energy efficiency maximization is also considered in [5], [17]. Another approach based on two-timescale hybrid precoding is studied in [22], [23]. In the referenced works, the analog precoder is designed by adapting to the channelstate-information (CSI) statistics, whereas the digital precoder is updated based on the effective CSI. However, in this paper, our aim is to design hybrid precoders by spectral efficiency maximization [3], [12].

In general, the problem of maximizing the SE is intractable for the joint-optimization over digital and analog precoders. To that end, it is shown in [3] that, instead of maximizing the SE, the optimal precoders can be found by minimizing the Euclidean distance between the fully digital and the hybrid precoders. This problem can be viewed as a CMCMF problem. The analog precoder is designed based on a codebook, using sparse signal processing techniques based on the orthogonal matching pursuit (OMP) [26], [27] concept. This approach developed a low complexity solution albeit it has poor performance in several cases [5]. Their performance is generally good under low-rank channels and when the hybrid transceivers are employed with more number of RF chains than the number of transmitted streams. To achieve better performance, some alternative works exist in the literature that improves performance by considering a codebook free design. For example, in [12] codebook free transceivers are proposed for different hybrid architectures, resulting in significantly improved performance as compared to codebook based approach. The optimal hybrid precoders were designed based on the Euclidean norm approximation. For simplicity, we follow the Frobenius norm approximation method, as well.

In [12], two algorithms are proposed namely, MO-ALTMIN and PE-ALTMIN for mmWave MIMO systems to solve the CMCMF problem using alternating minimization. Alternating minimization is a widely accepted tool for solving constrained matrix factorization problems [28]-[32]. Although, alternating minimization is very successful in dealing with such problems, proving convergence of these algorithms depends upon the specific problem under consideration. Herein, we have a nonconvex constrained version of the problem, which imposes additional challenges. The MO-ALTMIN algorithm uses sophisticated manifold optimization techniques to solve the problem, whereas the PE-ALTMIN algorithm imposes unitary constraints on the digital baseband precoder. Although the MO-ALTMIN algorithm does not require unitary constraints on the digital precoder, its scalability for larger system dimension is an issue due to the large computational complexity. The referenced work also proposed an algorithm for the partially connected case namely, SDR-ALTMIN, which is again based on alternating minimization between digital and analog precoders. The algorithm solves the non-convex quadratically constrained quadratic program (QCQP) using semidefinite relaxation to obtain the digital precoder, which is further limited by its computational complexity for larger system dimensions and its implementation becomes impractical.

\section{B. Contributions}

Our work is motivated by the problem of providing low complexity algorithms without imposing additional constraints on the design of digital and analog precoders. In particular, we develop algorithms to solve the CMCMF problem for largescale antenna arrays where solutions like MO-ALTMIN and SDR-ALTMIN become impractical. One of the challenges in solving hybrid transceivers design problems is the unitmodulus constraints. The unit-modulus constraints also appear in the context of radar sequence design [33] and the MM based algorithms are proposed in the literature to solve different problems [33], [34]. However, the existing algorithms in the literature can not be directly applied to the CMCMF problem due to fundamental differences in the problem formulation and constraint characterization. Our main contributions are:

- We propose efficient low-complexity algorithms for the fully connected and the partially connected hybrid architectures. For the fully-connected case, the hybrid A/D precoding problem is solved using a combination of alternating minimization and the majorization-minimization framework, whereas for the partially connected case the problem is solved using variable elimination and the minorization-maximization framework. First, the objective function is concentrated with respect to the digital precoder and it is eliminated from the problem. Then, the resulting objective is optimized first for the analog precoder using the MM framework. Once the concentrated objective converges to a stationary point, the digital precoder is obtained using the closed-form solution.

- Convergence to a stationary point of the iterates obtained from both the algorithms is established.

- We analyze the performance of the proposed algorithms by comparing the objective function value achieved with the number of iterations and the central processing unit (CPU) time, and a comparison of the SE with SNR and the number of RF chains is also presented. It is observed that the proposed algorithms converge faster to a stationary point as compared to the existing work in [12]. Results demonstrating the scalability of the algorithms are also shown.

Remark 1. It is important to note that the hybrid postcoding design problem at the receiver is similar to the one at the transmitter with a change in matrix dimensions. Thus, we also employ the proposed algorithms at the receiver side.

\section{Organization of the Paper}

The remainder of the paper is organized as follows. In Section II, the system model is described and the problem is 
formulated. In Section III, an algorithm to design the hybrid precoders for the fully connected architecture is presented, followed by its convergence analysis. Section IV presents the algorithm design for the partially connected architecture, followed by a proof of convergence. Simulation results are presented in Section V and Section VI concludes the work.

\section{Notations Used}

The following notations are used throughout the paper. A vector and a matrix are represented by $\mathbf{a}$ and $\mathbf{A}$ respectively. The $i, j$ element of a matrix is denoted as $\mathbf{A}(i, j)$. The complex exponential operation on each entry of a matrix is represented as $e^{(j \mathbf{A})}$, the phase/argument of each element of a matrix is denoted as $\arg (\mathbf{A})$. The trace operator and the Frobenius norm are represented as $\operatorname{Tr}(\mathbf{A})$ and $\|\mathbf{A}\|_{F} ;\|\mathbf{a}\|_{2}$ denotes the $\ell_{2}$-norm of the vector. The real part of a scalar complex variable $z$ or a matrix variable $\mathbf{Z}$, is represented as $\operatorname{Re}(z)$ or $\operatorname{Re}(\mathbf{Z})$, respectively. The symbol $|\cdot|$ denotes the modulus of a complex number. The Hermitian operation, conjugate, and transpose of a matrix are denoted as $\mathbf{A}^{H}, \mathbf{A}^{*}$, and $\mathbf{A}^{T}$ respectively. The Schur-Hadamard product between two matrices is represented as $\mathbf{A} \circ \mathbf{B} ; \mathbf{A} \succeq 0$ denotes a positive semi-definite (PSD) matrix. The set of Hermitian positive semi-definite matrices is represented as $\mathbb{S}_{n}^{+}$. The expectation operator is denoted as $\mathbb{E}(\cdot)$.

Note that, throughout this paper, we use the term MM interchangeably for denoting both the majorization-minimization and the minorization-maximization frameworks.

\section{System Model AND PROBlem Formulation}

In this section, first, we consider a system model for the point-to-point single user mmWave MIMO system and then, present the problem formulation.

\section{A. System Model}

Let us assume a single user MIMO system with $R_{x}$ receive and $T_{x}$ transmit antennas. The transmitter is equipped with $N_{t}<<T_{x}$ RF chains while $N_{r} \quad<R_{x}$ RF chains are on the receiver's side. The system is designed to transmit $N_{s} \leq \min \left\{N_{t}, N_{r}\right\}$ streams. For this reason, a hybrid precoder $\mathbf{P}_{t}=\mathbf{A}_{t} \mathbf{D}_{t}$ with dimensions $T_{x} \times N_{s}$ is employed at the transmitter, thus, the transmitted signal is written as $\mathbf{x}=\mathbf{A}_{t} \mathbf{D}_{t} \mathbf{s}$, where $\mathbf{s} \in \mathbb{C}^{N_{s} \times 1}$ is the transmitted symbol vector, $\mathbf{A}_{t} \in \mathbb{C}^{T_{x} \times N_{t}}$ is the analog precoding matrix, and $\mathbf{D}_{t} \in \mathbb{C}^{N_{t} \times N_{s}}$ is the lower-dimensional baseband (BB) digital precoding matrix. On similar lines, a hybrid postcoding operation with matrix $\mathbf{P}_{r}=\mathbf{A}_{r} \mathbf{D}_{r}$ with dimensions $R_{x} \times N_{s}$ is performed at the receiver, where $\mathbf{A}_{r} \in \mathbb{C}^{R_{x} \times N_{r}}$ is the analog postcoding matrix, and $\mathbf{D}_{r} \in \mathbb{C}^{N_{r} \times N_{s}}$ is the digital BB postcoding matrix.

The total transmit power constraint is given by $\left\|\mathbf{A}_{t} \mathbf{D}_{t}\right\|_{F}^{2}=$ $N_{s}$. The analog pre/post-coding matrices map the signals from the RF chains to the transmit/receive antennas using the phase-shifting networks. Therefore, every phase shifter in the network should satisfy unit-modulus constraints, which is translated as a constraint on each entry of the analog pre/postcoding matrices, that is, $\left|\mathbf{A}_{t}(i, j)\right|=1,\left|\mathbf{A}_{r}(i, j)\right|=1$, for all $i, j$. We consider the two most common hybrid architectures namely, the fully-connected and partially-connected ones. In the former, the signal from each RF chain is connected to all the antennas via the phase-shifting network, whereas in latter, the signal from each RF chain is connected to a subset of antennas [3], [35]. The total number of phase shifters required to implement a fully connected architecture on the transceiver sides are $T_{x} N_{t}$ and $R_{x} N_{r}$, respectively. For the partially connected case, each RF chain is connected to $T_{x} / N_{t}$ antenna on the transmitter side and $R_{x} / N_{r}$ antennas on the receiver side, as shown below,

$$
\mathbf{A}_{t}=\left[\begin{array}{cccc}
\mathbf{a}_{1} & \mathbf{0} & \ldots & \mathbf{0} \\
\mathbf{0} & \mathbf{a}_{2} & \ldots & \mathbf{0} \\
\vdots & & \ddots & \mathbf{0} \\
\mathbf{0} & \mathbf{0} & \ldots & \mathbf{a}_{N_{t}}
\end{array}\right]
$$

where each $\mathbf{a}_{i}$ is $T_{x} / N_{t} \times 1$ dimensional vector consisting unitmodulus entries. Therefore, this architecture requires fewer phase shifters compared to the fully connected architecture, but at the expense of loss in spectral efficiency. Correspondingly, the matrix $\mathbf{A}_{r}$ can be written in a similar manner having blockdiagonal structure, with each block of size $R_{x} / N_{r} \times 1$.

Considering a narrow-band block-fading propagation channel, the signal received at the receiver before and after the post-coding operation is given by,

$$
\begin{aligned}
\mathbf{y} & =\mathbf{H P}_{t} \mathbf{s}+\mathbf{n}, \\
\mathbf{y}_{r} & =\mathbf{P}_{r}^{H} \mathbf{y}
\end{aligned}
$$

where $\mathbf{H} \in \mathbb{C}^{R_{x} \times T_{x}}$ is the channel matrix, and $\mathbf{n} \in \mathbb{C}^{R_{x} \times 1}$ is independent identically distributed (i.i.d) circular-symmetric complex Gaussian noise, $\mathcal{C N}\left(\mathbf{0}, \sigma_{n}^{2} \mathbf{I}\right), \sigma_{n}^{2}$ represents the noise variance. Considering the symbol vector to be zero-mean, with i.i.d entries, then its second order statistical characterization is, $\mathbb{E}\left\{\mathbf{s s}^{H}\right\}=\sigma_{s}^{2} \mathbf{I}$, where $\sigma_{s}^{2}$ is the variance of $s_{i}, \forall i$. The SE of such a system is given as,

$$
\mathrm{SE}=\log _{2} \operatorname{det}\left(\mathbf{I}+\mathbf{R}_{n}^{-1} \mathbf{P}_{r}^{H} \mathbf{H} \mathbf{P}_{t} \mathbf{P}_{t}^{H} \mathbf{H}^{H} \mathbf{P}_{r}\right),
$$

where $\mathbf{R}_{n}=\sigma_{n}^{2} \mathbf{P}_{r}^{H} \mathbf{P}_{r}$ and we have assumed that the input symbol vector $\mathbf{s}$ follows a Gaussian circular symmetric distribution [5], with $\sigma_{s}^{2}=1$.

\section{B. Channel Model}

A narrowband clustered channel is considered based on the Saleh-Valenzuela model, which accurately captures the characteristics of a typical mmWave channel including severe path loss, limited scattering, high correlation among antennas due to closely packed antenna arrays, etc., [36], [37]. Assuming a total number of $N_{p}$ propagation paths between the transceiver's ends, the channel is modeled by,

$$
\mathbf{H}=\sqrt{\frac{T_{x} R_{x}}{N_{p}}} \sum_{k=1}^{N_{p}} \alpha_{k} \mathbf{b}_{r}\left(\phi_{k}^{r}\right) \mathbf{b}_{t}\left(\phi_{k}^{t}\right)^{H},
$$

where $\mathbf{b}_{r}\left(\phi_{k}^{r}\right)$ and $\mathbf{b}_{t}\left(\phi_{k}^{t}\right)$ represents the normalized array response or steering vectors associated with receive and transmit antenna arrays, $\alpha_{k} \in \mathbb{C}$ is the gain along the $k$-th path, $\phi_{k}^{r}$ and $\phi_{k}^{t}$ are the azimuth angle of arrival and departure, respectively 
for the $k$-th path. Assuming a uniform linear array (ULA) of isotropic antenna elements at the transmitter and at the receiver, then the steering vector in a direction $\phi$ is given by,

$$
\mathbf{b}(\phi)=\sqrt{\frac{1}{M}}\left[1, e^{j k d \sin (\phi)}, \ldots, e^{j(M-1) k d \sin (\phi)}\right]
$$

where $k=\frac{2 \pi}{\lambda}$ is the wavenumber, $\lambda$ is the carrier wavelength, $M$ is the number of antenna elements in the array, and $d$ is the inter-element spacing between two antennas.

\section{Problem Formulation}

The problem is to find the optimal hybrid precoder and postcoder by maximizing the system SE as shown in (4) by solving the following optimization problem,

$$
\begin{array}{cl}
\mathcal{P}_{1}: \max _{\mathbf{A}_{t}, \mathbf{D}_{t}, \mathbf{A}_{r}, \mathbf{D}_{r}} & \operatorname{SE}\left(\mathbf{A}_{t}, \mathbf{D}_{t}, \mathbf{A}_{r}, \mathbf{D}_{r}\right) \\
\text { subject to } & \mathbf{A}_{t} \in \chi_{t}, \mathbf{A}_{r} \in \chi_{r} \\
& \left\|\mathbf{A}_{t} \mathbf{D}_{t}\right\|_{F}^{2}=N_{s},
\end{array}
$$

where $\chi_{t}$ and $\chi_{r}$ are the sets imposing power, unit-modulus and structural constraints on the design of analog precoder and postcoder, respectively. Problem $\mathcal{P}_{1}$ is intractable due to the nonconvexity of the objective function and the constraints. Even for the fully digital scenario, it is difficult to solve this problem [38]. For this reason, the most commonly used approach is to decouple the designs at the transmitter's and the receiver's side [3]. Thus, instead of maximizing the SE, the optimal hybrid precoders are designed by maximizing the mutual information achieved with Gaussian signaling [3]. Therefore, the optimization problem is given by,

$$
\begin{array}{cl}
\mathcal{P}_{2}: \max _{\mathbf{A}_{t}, \mathbf{D}_{t}} & \log _{2} \operatorname{det}\left(\mathbf{I}+\sigma_{n}^{-2} \mathbf{H} \mathbf{A}_{t} \mathbf{D}_{t} \mathbf{D}_{t}^{H} \mathbf{A}_{t}^{H} \mathbf{H}^{H}\right) \\
\text { subject to } & \mathbf{A}_{t} \in \chi_{t} \\
& \left\|\mathbf{A}_{t} \mathbf{D}_{t}\right\|_{F}^{2}=N_{s} .
\end{array}
$$

It is easy to see that problem $\mathcal{P}_{2}$ is still nonconvex and difficult to solve. In order to solve this problem, it has been shown in [3] that the optimal precoders can be equivalently found by minimizing the Euclidean distance between the fully digital and the hybrid precoders,

$$
\begin{aligned}
\mathcal{P}_{3}: \min _{\substack{\mathbf{A}_{t}, \mathbf{D}_{t} \\
\text { subject to }}}\left\|\mathbf{P}_{t} \in \mathbf{A}_{t} \mathbf{D}_{t}\right\|_{F}^{2} \\
\left\|\mathbf{A}_{t} \mathbf{D}_{t}\right\|_{F}^{2}=N_{s} .
\end{aligned}
$$

Problem $\mathcal{P}_{3}$ is equivalent to finding the projection of the fully digital precoder onto the set of hybrid precoders. Equivalently it can also be considered as a CMCMF problem. We will revisit this problem later and provide efficient solutions under different hybrid architectures.

For the receiver, the minimum mean square error (MMSE) based criterion is employed,

$$
\begin{gathered}
\mathcal{P}_{4}: \min _{\substack{\mathbf{A}_{r}, \mathbf{D}_{r} \\
\text { subject to } \mathbf{A}_{r} \in \chi_{r} .}} \mathbb{E}\left\|\mathbf{s}-\mathbf{D}_{r}^{H} \mathbf{A}_{r}^{H} \mathbf{y}\right\|_{2}^{2} \\
\end{gathered}
$$

Problem $\mathcal{P}_{4}$ is also nonconvex. An equivalent formulation of this problem as shown in [3] is given by,

$$
\begin{aligned}
\mathcal{P}_{5}: & \min _{\substack{\mathbf{A}_{r}, \mathbf{D}_{r} \\
\text { subject to } \mathbf{A}_{r} \in \chi_{r},}}\left\|\mathbb{E}\left\{\mathbf{y y}^{H}\right\}^{\frac{1}{2}}\left(\mathbf{P}_{r}-\mathbf{A}_{r} \mathbf{D}_{r}\right)\right\|_{F}^{2} \\
&
\end{aligned}
$$

where $\mathbb{E}\left\{\mathbf{y} \mathbf{y}^{H}\right\}^{\frac{1}{2}}$ is the positive square root of the covariance matrix $\mathbb{E}\left\{\mathbf{y y} \mathbf{y}^{H}\right\}$. Problem $\mathcal{P}_{5}$ has a similar mathematical structure to that of problem $\mathcal{P}_{3}$. The only difference here is that instead of standard Frobenius norm, the projection is with respect to the $\mathbb{E}\left\{\mathbf{y} \mathbf{y}^{H}\right\}^{\frac{1}{2}}$-weighted Frobenius norm. However, this weight does not affect the matrix factorization structure of the problem and the algorithms developed herein are applicable to both problems of precoder as well as postcoder designs.

Therefore, we primarily focus on the precoder design and apply the same algorithms for the postcoder design. For notational convenience, from now onwards we drop the subscript $(\cdot)_{t}$ associated with the digital and the hybrid precoders. We formulate the following optimization problem,

$$
\begin{gathered}
\mathcal{P}_{6}: \min _{\mathbf{A}, \mathbf{D}}\|\mathbf{P}-\mathbf{A D}\|_{F}^{2} \\
\text { subject to } \mathbf{A} \in \mathcal{A} \\
\|\mathbf{A D}\|_{F}^{2}=N_{s},
\end{gathered}
$$

where $\mathbf{P}, \mathbf{A}$, and $\mathbf{D}$ denotes the fully digital, analog, and BB digital precoding matrices. The set $\mathcal{A}$ introduces structural constraints on the design of analog precoding matrix in different hybrid architectures. In this problem, we basically try to approximate the beam-response obtained by a fully digital precoding matrix with a hybrid precoding structure. For a single user MIMO system, the unconstrained fully digital precoder is obtained from the singular value decomposition (SVD) of the channel matrix and the water-filling algorithm [39].

The problem $\mathcal{P}_{6}$ is a CCMMF one. Our main objective in this paper is to provide efficient algorithms to solve $\mathcal{P}_{6}$ with guaranteed convergence under different hybrid architectures. In the subsequent sections, we consider both the fully connected as well as the partially connected architectures and propose efficient algorithms to solve the problem with provable convergence to a stationary point.

\section{MAJORIZATION-MINIMIZATION BASED HYBRID} Precoding For the Fully CONNECTED ARChitecture

In a fully-connected architecture, each RF chain is connected to all the antennas, which imposes a unit-modulus constraint on each entry of the analog precoding matrix $\mathbf{A}$. Therefore, the set $\mathcal{A}$ of problem $\mathcal{P}_{6}$ is given as, $\mathcal{A}=\{\mathbf{A} \in$ $\left.\left.\mathbb{C}^{T_{x} \times N_{t}}|| \mathbf{A}(i, j)\right|^{2}=1,1 \leq i \leq T_{x}, 1 \leq j \leq N_{t}\right\}$. For the receiver side a similar set is constructed by replacing $T_{x}$ with $R_{x}$ and $N_{t}$ with $N_{r}$. It is important to note that here, we have assumed a squared constant modulus constraint instead of constant modulus constraint, to alleviate the differentiability issues associated with the constant modulus function. This small change does not introduce ambiguities and will help 
later while deriving the KKT conditions to prove convergence of the proposed algorithm.

It is important to note that, problem $\mathcal{P}_{6}$ is intractable because the objective function is nonconvex in variables $\mathbf{A}$ and $\mathbf{D}$, jointly. Moreover, the constraints are also nonconvex, which further complicates the problem. It can be seen that the objective function is convex in one variable given the other. Therefore, we adopt an alternating minimization approach to solve the problem [31]. The idea behind alternating minimization is to exploit partially convexity in the optimization problem and split the joint optimization problem into several subproblems depending upon the number of unknown variables. In other words, it iteratively minimizes the objective function in one variable by fixing the remaining variables, and viceversa. This procedure is repeated for all the variables until some convergence criterion is satisfied. In our case, although the objective function of problem $\mathcal{P}_{6}$ is partially convex in $\mathbf{A}$ and $\mathbf{D}$, the constraints are still nonconvex. To tackle this, we consider the problem without the power constraint and then normalize the digital precoder with an appropriate scaling factor [12]. We first consider the optimization problem over $\mathbf{A}$ provided $\mathbf{D}=\mathbf{D}_{l}$, where $\mathbf{D}_{l}$ is an estimate of $\mathbf{D}$ available at iteration $l$. For this sub-problem, we still have the nonconvex unit-modulus constraints on the entries of matrix A. Therefore, we propose to use the majorization-minimization framework, which leads to a very simple algorithm to compute the analog precoder.

Before proceeding further, we now briefly introduce the basic idea behind the majorization-minimization framework to solve minimization problems and then, propose an algorithmic solution to solve problem $\mathcal{P}_{6}$.

\section{A. MM framework}

The majorization-minimization method works on the philosophy of iteratively solving a sequence of easier problems [33], [40], [41]. For example, consider the problem of minimizing a function $f(\mathbf{x})$ over set $\mathbf{x} \in \mathcal{G}$. The direct minimization of the function in general can be difficult, specifically when the objective and/or constraints are nonconvex. Thus, instead of directly minimizing the function $f(\mathbf{x})$, a surrogate function $\tilde{f}\left(\mathbf{x} ; \mathbf{x}_{k}\right)$ majorizing the original objective function $f(\mathbf{x})$ is minimized at the $k$-th iteration. A valid surrogate function for the minimization problem has the following properties,

$$
\begin{aligned}
\tilde{f}\left(\mathbf{x} ; \mathbf{x}_{k}\right) & >f(\mathbf{x}), \forall \mathbf{x} \in \mathcal{G} \\
\tilde{f}\left(\mathbf{x}_{k} ; \mathbf{x}_{k}\right) & =f\left(\mathbf{x}_{k}\right) \\
\nabla \tilde{f}\left(\mathbf{x}_{k} ; \mathbf{x}_{k}\right) & =\nabla f\left(\mathbf{x}_{k}\right) .
\end{aligned}
$$

The above properties indicate that the surrogate function should be a tight upper bound of the original objective function. Therefore, an algorithm based on this framework starts with a feasible initial-point $\mathbf{x}_{0} \in \mathcal{G}$ and iteratively minimizes the surrogate function,

$$
\mathbf{x}_{k+1} \in \arg \min _{\mathbf{x} \in \mathcal{G}} \tilde{f}\left(\mathbf{x} ; \mathbf{x}_{k}\right) .
$$

Because of the tight upper bound property of the surrogate function, at every step, the objective function value is decreased and the sequence of iterates $\left\{\mathbf{x}_{k}\right\}$ converges to a stationary point of the original problem. For brevity, the steps of the majorization-minimization method are summarized as follows,

1) Initialize $\mathrm{x}_{0}$ to a feasible point from the set $\mathcal{G}$.

2) Construct a majorizing function $\tilde{f}\left(\mathbf{x} ; \mathbf{x}_{k}\right)$ of the function $f(\mathbf{x})$ at $\mathbf{x}_{k}$ satisfying properties (13)-(15).

3) Update $\mathbf{x}_{k+1} \in \arg \min _{\mathbf{x} \in \mathcal{G}} \tilde{f}\left(\mathbf{x} ; \mathbf{x}_{k}\right)$.

4) If a convergence condition is met, then stop. Otherwise, increment $k$ to $k+1$ and go to step 2 .

For more detailed information on the MM framework, one may refer to [33], [40], [41] and references therein. Since, the surrogate function $\tilde{f}\left(\mathbf{x} ; \mathbf{x}_{k}\right)$ is a tight majorizer of the original function $f(\mathbf{x})$ and satisfies the properties (13) to (15), thereby, implying that the objective value at every iteration decreases monotonically. To formally prove the convergence of an $\mathrm{MM}$ algorithm, first, we introduce the first-order optimality condition for the minimization of a continuously differentiable function [42].

Proposition III.1. Consider $f: \mathbb{R}^{n} \rightarrow \mathbb{R}$ be a continuously differentiable function and if $\mathbf{x}_{\infty}$ is a local minimum of $f$ over a subset $\mathcal{G}$ of $\mathbb{R}^{n}$, then

$$
\nabla f\left(\mathbf{x}_{\infty}\right)^{T}\left(\mathbf{x}-\mathbf{x}_{\infty}\right) \geq 0, \forall \mathbf{x} \in \mathcal{G} .
$$

A vector $\mathbf{x}$ satisfying the optimality condition (17) is referred to as a stationary point. For more insights into the convergence properties of the MM framework, one may refer to [33], [40], [41] and references therein. To deal with maximization problems, the surrogate function should be a tight minorizer of the objective one, and this framework is known as the minorization-maximization.

\section{B. Majorization-Minimization Based Analog Precoder Design}

Based on the alternating minimization framework, the first step is to solve the problem for the analog precoder assuming a solution for the digital precoder. We rewrite the minimization problem $\mathcal{P}_{6}$ as,

$$
\begin{gathered}
\mathcal{P}_{7}: \min _{\mathbf{A}}\left\|\mathbf{P}-\mathbf{A D}_{l}\right\|_{F}^{2} \\
\text { subject to } \mathbf{A} \in \mathcal{A} .
\end{gathered}
$$

For the time being, we have omitted the power constraint in $\mathcal{P}_{7}$ and will be dealt with later in the next sub-section. Problem $\mathcal{P}_{7}$ is a nonconvex optimization problem because of the nonconvex unit-modulus constraints.

In order to solve problem $\mathcal{P}_{7}$ using the majorizationminimization framework, the first step is to find a majorizing function of the objective such that the overall problem is easily solved. We present the following lemma which is useful in the construction of the majorizing function [33], [34], [40], [41].

Lemma III.2. The quadratic function of the form $\mathbf{a}^{H} \mathbf{S a}$, with $\mathbf{S}$ being a PSD matrix is majorized by $\mathbf{a}^{H} \mathbf{T a}+$ $2 \operatorname{Re}\left(\mathbf{a}^{H}(\mathbf{S}-\mathbf{T}) \mathbf{a}_{k}\right)+\mathbf{a}_{k}^{H}(\mathbf{T}-\mathbf{S}) \mathbf{a}_{k}$ at the point $\mathbf{a}_{k}$, where $\mathbf{T}$ is a PSD matrix such that $\mathbf{T} \succeq \mathbf{S}$. 
Proof. The proof of the above argument is fairly straightforward. Consider the second order Taylor's expansion of the function $\mathbf{a}^{H} \mathbf{S a}$ around $\mathbf{a}_{k}$,

$$
\begin{aligned}
& \mathbf{a}^{H} \mathbf{S a}= \\
& \mathbf{a}_{k}^{H} \mathbf{S} \mathbf{a}_{k}+2 \operatorname{Re}\left(\mathbf{a}_{k}^{H} \mathbf{S}\left(\mathbf{a}-\mathbf{a}_{k}\right)\right)+\left(\mathbf{a}-\mathbf{a}_{k}\right)^{H} \mathbf{S}\left(\mathbf{a}-\mathbf{a}_{k}\right)
\end{aligned}
$$

Now, replacing the Hessian matrix $\mathbf{S}$ with a matrix $\mathbf{T}$ such that $\mathbf{T} \succeq \mathbf{S}$, this implies,

$$
\begin{aligned}
& \mathbf{a}^{H} \mathbf{S a} \leq \\
& \mathbf{a}_{k}^{H} \mathbf{S} \mathbf{a}_{k}+2 \operatorname{Re}\left(\mathbf{a}_{k}^{H} \mathbf{S}\left(\mathbf{a}-\mathbf{a}_{k}\right)\right)+\left(\mathbf{a}-\mathbf{a}_{k}\right)^{H} \mathbf{T}\left(\mathbf{a}-\mathbf{a}_{k}\right) .
\end{aligned}
$$

Upon rearranging the above equation and using a fact that $\operatorname{Re}(z)=\operatorname{Re}\left(z^{*}\right)$, we get,

$$
\begin{aligned}
& \mathbf{a}^{H} \mathbf{S a} \leq \\
& \quad \mathbf{a}^{H} \mathbf{T a}+2 \operatorname{Re}\left(\mathbf{a}^{H}(\mathbf{S}-\mathbf{T}) \mathbf{a}_{k}\right)+\mathbf{a}_{k}^{H}(\mathbf{T}-\mathbf{S}) \mathbf{a}_{k} .
\end{aligned}
$$

This concludes the proof.

For any twice differentiable function with bounded curvature, Lemma III.2 is also known by the name of quadratic upper bound principle [40]. First, we rewrite the objective using the row-wise decomposition of the matrix $\mathbf{P}$ and equivalence between the Frobenius and $l_{2}$ norms, as,

$$
\begin{gathered}
\mathcal{P}_{8}: \min _{\mathbf{A}} \sum_{i=1}^{T_{x}} \mathbf{p}_{i}^{H} \mathbf{p}_{i}-2 \operatorname{Re}\left(\mathbf{p}_{i}^{H} \mathbf{D}_{l}^{H} \mathbf{a}_{i}\right)+\mathbf{a}_{i}^{H} \mathbf{D}_{l} \mathbf{D}_{l}^{H} \mathbf{a}_{i} \\
\text { subject to } \mathbf{A} \in \mathcal{A},
\end{gathered}
$$

where $\mathbf{p}_{i}^{H}$ and $\mathbf{a}_{i}^{H}$ are the $i$-th rows of the matrices $\mathbf{P}$ and A, respectively. The last term in the objective function is the summation of $T_{x}$ convex functions, which is a convex function. To use the MM framework, each component function of the third term in the objective function is majorized using Lemma III.2, i.e.,

$$
\begin{array}{r}
\mathbf{a}_{i}^{H} \mathbf{S}_{l} \mathbf{a}_{i} \leq\left(\mathbf{a}_{i, k}\right)^{H} \mathbf{S}_{l} \mathbf{a}_{i, k}+2 \operatorname{Re}\left(\left(\mathbf{a}_{i}-\mathbf{a}_{i, k}\right) \mathbf{S}_{l} \mathbf{a}_{i, k}\right) \\
+\left(\mathbf{a}_{i}-\mathbf{a}_{i, k}\right)^{H} \mathbf{T}_{l}\left(\mathbf{a}_{i}-\mathbf{a}_{i, k}\right),
\end{array}
$$

where $\left(\mathbf{a}_{i, k}\right)^{H}$ is the iterate available at $k$-th iteration, $\mathbf{S}_{l}=$ $\mathbf{D}_{l} \mathbf{D}_{l}^{H}$, and $\mathbf{T}$ is a Hermitian positive semi-definite matrix such that, $\mathbf{T}_{l} \succeq \mathbf{S}_{l}$. Here, we choose $\mathbf{T}_{l}=\lambda_{\max }\left(\mathbf{S}_{l}\right) \mathbf{I}$, where $\lambda_{\max }\left(\mathbf{S}_{l}\right)$ is the maximum eigenvalue of the matrix $\mathbf{S}_{l}$. Then, we get,

$$
\begin{aligned}
\mathbf{a}_{i}^{H} \mathbf{S}_{l} \mathbf{a}_{i} \leq & \lambda_{\max }\left(\mathbf{S}_{l}\right) \mathbf{a}_{i}^{H} \mathbf{a}_{i}+2 \operatorname{Re}\left(\mathbf{a}_{i}^{H}\left(\mathbf{S}_{l}-\lambda_{\max }\left(\mathbf{S}_{l}\right) \mathbf{I}\right) \mathbf{a}_{i, k}\right) \\
& +\left(\mathbf{a}_{i, k}\right)^{H}\left(\lambda_{\max }\left(\mathbf{S}_{l}\right) \mathbf{I}-\mathbf{S}_{l}\right)\left(\mathbf{a}_{i, k}\right) .
\end{aligned}
$$

It is readily seen that the first term on the right side of the above inequality is just a constant, that is, $\mathbf{a}_{i}^{H} \mathbf{a}_{i}=N_{t}, \forall i$ and also the last term is independent of the variable $\mathbf{a}_{i}^{H}$. Thus,

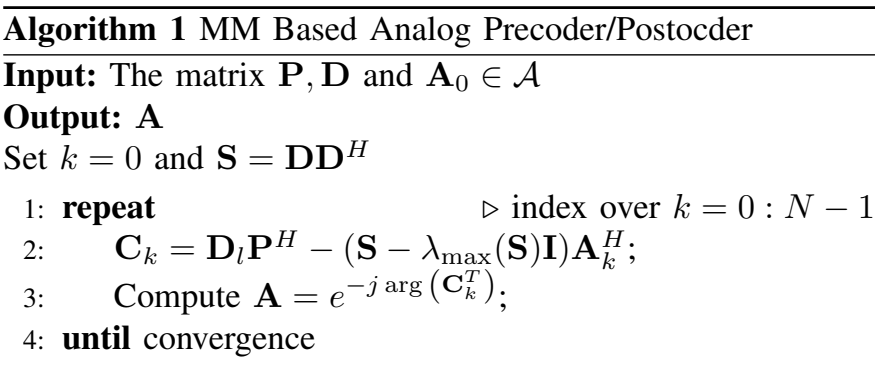

the objective function of problem $\mathcal{P}_{8}$ by using the surrogate function from (21) is replaced as,

$$
\begin{aligned}
f\left(\mathbf{A} ; \mathbf{D}_{l}\right)= & \sum_{i=1}^{T_{x}} \mathbf{p}_{i}^{H} \mathbf{p}_{i}-2 \operatorname{Re}\left(\mathbf{p}_{i}^{H} \mathbf{D}_{l}^{H} \mathbf{a}_{i}\right)+\mathbf{a}_{i}^{H} \mathbf{D}_{l} \mathbf{D}_{l}^{H} \mathbf{a}_{i} \\
\tilde{f}\left(\mathbf{A} ; \mathbf{A}_{k}, \mathbf{D}_{l}\right)= & \sum_{i=1}^{T_{x}} \mathbf{p}_{i}^{H} \mathbf{p}_{i}-2 \operatorname{Re}\left(\mathbf{p}_{i}^{H} \mathbf{D}_{l}^{H} \mathbf{a}_{i}\right) \\
& +\lambda_{\max }\left(\mathbf{S}_{l}\right) \mathbf{a}_{i}^{H} \mathbf{a}_{i} \\
& +2 \operatorname{Re}\left(\mathbf{a}_{i}^{H}\left(\mathbf{S}_{l}-\lambda_{\max }\left(\mathbf{S}_{l}\right) \mathbf{I}\right) \mathbf{a}_{i, k}\right) \\
& +\left(\mathbf{a}_{i, k}\right)^{H}\left(\lambda_{\max }\left(\mathbf{S}_{l}\right) \mathbf{I}-\mathbf{S}_{l}\right)\left(\mathbf{a}_{i, k}\right),
\end{aligned}
$$

It should be pointed out that the surrogate function $\tilde{f}\left(\mathbf{A} ; \mathbf{A}_{k}, \mathbf{D}_{l}\right)$ has the following properties,

$$
\begin{aligned}
\tilde{f}\left(\mathbf{A} ; \mathbf{A}_{k}, \mathbf{D}_{l}\right) & \geq f\left(\mathbf{A} ; \mathbf{D}_{l}\right), \forall \in \mathcal{A}, \\
\tilde{f}\left(\mathbf{A}_{k} ; \mathbf{A}_{k}, \mathbf{D}_{l}\right) & =f\left(\mathbf{A}_{k} ; \mathbf{D}_{l}\right), \\
\nabla_{\mathbf{A}} \tilde{f}\left(\mathbf{A}_{k} ; \mathbf{A}_{k}, \mathbf{D}_{l}\right) & =\nabla_{\mathbf{A}} f\left(\mathbf{A}_{k} ; \mathbf{D}_{l}\right) .
\end{aligned}
$$

Therefore, the function $\tilde{f}\left(\mathbf{A} ; \mathbf{A}_{k}, \mathbf{D}_{l}\right)$ is a valid majorizer of the function $f\left(\mathbf{A} ; \mathbf{D}_{l}\right)$ [33], [40], [41]. Now, using the above surrogate function and adopting the MM framework we solve the problem $\mathcal{P}_{8}$ iteratively,

$$
\begin{gathered}
\mathcal{P}_{9}: \min _{\mathbf{A}} \tilde{f}\left(\mathbf{A} ; \mathbf{A}_{k}, \mathbf{D}_{l}\right) \\
\text { subject to } \mathbf{A} \in \mathcal{A} .
\end{gathered}
$$

After ignoring the constant terms in problem $\mathcal{P}_{9}$ we arrive at the following projection problem,

$$
\begin{gathered}
\mathcal{P}_{10}: \min _{\mathbf{A}} \sum_{i=1}^{T_{x}}\left\|\mathbf{a}_{i}-\mathbf{c}_{i, k}\right\|_{2}^{2} \\
\text { subject to } \mathbf{A} \in \mathcal{A},
\end{gathered}
$$

where $\mathbf{c}_{i, k}=\left[\mathbf{D}_{l} \mathbf{p}_{i}-\left(\mathbf{S}-\lambda_{\max }(\mathbf{S}) \mathbf{I}\right) \mathbf{a}_{i, k}\right]$. It is straightforward to see that the above problem admits the following closed-form solution,

$$
\mathbf{a}_{i}=e^{j \arg \left(\mathbf{c}_{i, k}\right)}, \forall i
$$

and the update for the complete matrix $\mathbf{A}$ is given by,

$$
\mathbf{A}=e^{-j \arg \left(\mathbf{C}_{k}^{T}\right)},
$$

where $\mathbf{C}_{k}=\mathbf{D}_{l} \mathbf{P}^{H}-\left(\mathbf{S}-\lambda_{\max }(\mathbf{S}) \mathbf{I}\right) \mathbf{A}_{k}^{H}$. Now, for the sake of convenience, we summarize the overall algorithm and it is given in Algorithm 1. 


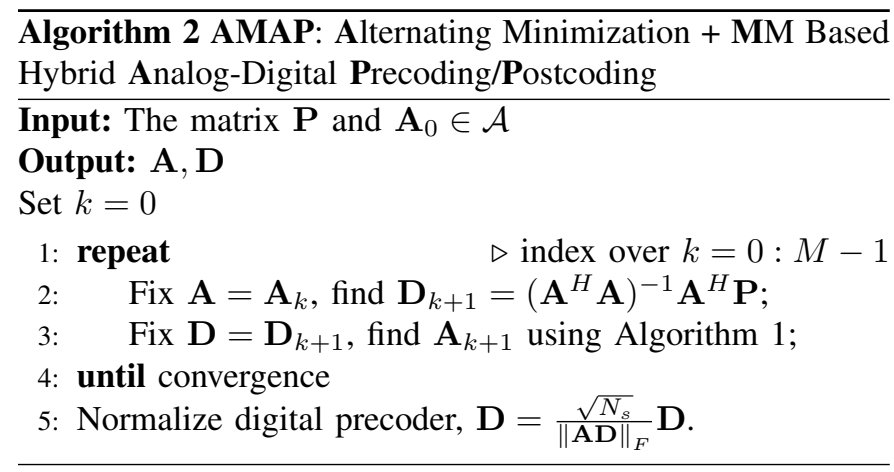

\section{Digital Precoder Design}

Now, we focus on the design of digital BB precoding matrix, $\mathbf{D}$ provided, $\mathbf{A}$ is given. Thus, we formulate the following problem,

$$
\begin{aligned}
& \mathcal{P}_{11}: \min _{\mathbf{D}}\left\|\mathbf{P}-\mathbf{A}_{l} \mathbf{D}\right\|_{F}^{2} \\
& \text { subject to }\left\|\mathbf{A}_{l} \mathbf{D}\right\|_{F}^{2}=N_{s},
\end{aligned}
$$

where, $\mathbf{A}_{l}$ is an estimate of the matrix $\mathbf{A}$ available at $l$-th iteration. It is easy to see that above the problem is a nonconvex QCQP, because of the convex equality constraint. To solve problem $\mathcal{P}_{10}$, one approach is to relax the equality constraint with inequality, then, it becomes a convex minimization problem, and can be efficiently solved using the solvers like CVX, SeduMi. But we want to avoid this route, as it incurs huge computation burden for larger systems. One can also solve the unconstrained problem for $\mathbf{D}$, provided $\mathbf{P}$ satisfies the power constraint. In such a case, it is readily seen that the total power constraint on $\mathbf{P}=\mathbf{A D}$ is always satisfied, that is $\|\mathbf{A D}\|_{F}^{2}=\left\|\Pi_{\mathbf{A}} \mathbf{P}\right\|_{F}^{2} \leq N_{s}$, provided $\mathbf{P}$ satisfies $\|\mathbf{P}\|_{F}^{2} \leq N_{s}$ where $\Pi_{\mathbf{A}}=\mathbf{A}\left(\mathbf{A}^{H} \mathbf{A}\right)^{-1} \mathbf{A}^{H}$ is the orthogonal projection operator of the matrix $\mathbf{A}$. But this is not the optimal solution, because the power constraint is satisfied with inequality.

In order to satisfy the power constraint with equality, one can simply solve the unconstrained version of the problem $\mathcal{P}_{11}$ as,

$$
\mathbf{D}=\left(\mathbf{A}_{l}^{H} \mathbf{A}_{l}\right)^{-1} \mathbf{A}_{l}^{H} \mathbf{P},
$$

where the inverse $\left(\mathbf{A}_{l}^{H} \mathbf{A}_{l}\right)^{-1}$ is guaranteed to exist because $\mathbf{A}_{l}$ is a full-column rank matrix, as $T_{x}>>N_{t}$. Then, normalize the digital precoder by a factor of $\frac{\sqrt{N_{s}}}{\|\mathbf{A D}\|_{F}}$. An insight from this normalization comes from the fact that as long as the Euclidean distance between the optimal fully digital precoder and the hybrid precoder without equality constraint is small, that is if the estimate obtained from the closed-form solution is consistent, one can also achieve a small distance with this normalization [12].

The complete algorithm to design the hybrid precoder from the fully digital precoder, henceforth referred to as AMAP (Alternating Minimization + MM Based Hybrid AnalogDigital Precoding/Postcoding), is presented in Algorithm 2. For the postcoder design, the digital BB postocder is obtained without scaling factor $\frac{\sqrt{N_{s}}}{\|\mathbf{A D}\|_{F}}$ appearing AMAP algorithm.

\section{Convergence Analysis of Algorithm AMAP}

In this section, we provide an outline of the convergence proof. This algorithm uses a combination of the MM and the alternating minimization framework [43]. The MM method iteratively minimizes the upper bound of the objective function to solve the nonconvex analog precoding problem.

Theorem III.3. The solution sequence obtained from algorithm AMAP converges to the set of $K K T$ points $(\mathcal{K})$ of problem $\mathcal{P}_{3}$, excluding the power constraint and the corresponding scaling in step 5 of the algorithm.

Proof. See Appendix A.

\section{E. Complexity Analysis of Algorithm AMAP}

Algorithm 2 uses alternating minimization, to compute the analog and digital precoding matrices. In each step of the MM algorithm, we need to carry out matrix multiplications and the number of floating-point operations (FLOPS) required to compute one matrix multiplication $\mathbf{X} \times \mathbf{Z}$ are $(m p(2 n-1))$, where $\mathbf{X}$ and $\mathbf{Z}$ are the matrices with dimensions $m \times n$ and $n \times p$, respectively. Here we investigate the worstcase complexity of the algorithms. Considering the naive implementation of matrix multiplications, we first analyze the complexity of Algorithm 1. The complexity of carrying out the multiplication $\mathbf{A}_{k} \mathbf{S}$ is $O\left(T_{x} N_{t}^{2}\right)$, for $\mathbf{P} \mathbf{D}^{H}$ it is $O\left(T_{x} N_{s} N_{t}\right)$. The complexity of carrying out the exponential operation is $O\left(T_{x} N_{t}\right)$. Thus, the worst-case complexity of Algorithm 1 is $O\left(T_{x} N_{t}^{2}\right)$ per iteration. Apart from this, the maximum eigenvalue of Hermitian positive semidefinite matrix $\mathbf{S}$ needs to be computed, which is easy to compute even for large matrices using Krylov-Schur Algorithm [44]. Thus, our algorithm is computationally efficient. Similarly, the worst-case complexity of the AMAP algorithm is $O\left(T_{x}^{3}\right)$ per iteration. This can be improved provided matrix multiplications and inverses carried out efficiently. To compute the digital precoder, both algorithms use the same update step to compute pseudoinverse with $O\left(T_{x}^{3}\right)$ operations.

\section{HYBRID PRECODING FOR THE PARTIALly CONNECTED ARCHITECTURE}

In a partially-connected architecture each RF chain is connected to $T_{x} / N_{t} \times 1$ antenna elements as shown in (1). The CMCMF problem for this architecture is given by,

$$
\begin{aligned}
\mathcal{P}_{12}: & \min _{\substack{\mathbf{A}, \mathbf{D} \\
\text { subject to }}} \| \mathbf{A} \in \mathbf{A} \in \mathcal{C}, \\
\|\mathbf{A D}\|_{F}^{2} & =N_{s},
\end{aligned}
$$

where the set $\mathcal{C}$ contains the block-matrices of the form and the dimensions presented in equation (1), with each block having constant modulus entries. Due to this structural constraint on the analog precoding matrix $\mathbf{A}$, the power constraint can 
be significantly simplified as, $\|\mathbf{A D}\|_{F}^{2}=\|\mathbf{D}\|_{F}^{2}=\frac{N_{s} N_{t}}{T_{x}}$. Therefore, problem $\mathcal{P}_{12}$ can be written as,

$$
\begin{aligned}
\mathcal{P}_{13}: & \min _{\substack{\mathbf{A}, \mathbf{D} \\
\text { subject to }}}\|\mathbf{A} \in \mathcal{P}-\mathbf{A D}\|_{F}^{2} \\
& \|\mathbf{D}\|_{F}^{2}=\frac{N_{s} N_{t}}{T_{x}}=\beta .
\end{aligned}
$$

Problem $\mathcal{P}_{13}$ is a nonconvex optimization problem because neither the objective nor the constraints are convex. Problem $\mathcal{P}_{13}$ is solved using a combination of variable elimination and the MM framework, which leads to a much simpler and efficient solution compared to the one for the fully connected case.

\section{A. Derivation of the Algorithmic Solution}

The basic idea behind variable elimination is to concentrate the objective function with respect to one of the parameters and substitute back its closed-form solution in the objective, to obtain the problem in terms of only one variable. This approach significantly simplifies the objective in some cases, where the back substitution does not make the problem illposed.

In order to solve problem $\mathcal{P}_{13}$, we first concentrate the objective with respect to $\mathbf{D}$ assuming $\mathbf{A}$ is given,

$$
\begin{array}{r}
\mathcal{P}_{14}: \min _{\mathbf{D}}\|\mathbf{P}-\mathbf{A D}\|_{F}^{2} \\
\text { subject to }\|\mathbf{D}\|_{F}^{2}=\beta .
\end{array}
$$

Upon expanding the objective function we have,

$$
\begin{gathered}
\mathcal{P}_{15}: \min _{\mathbf{D}} \operatorname{Tr}\left(\mathbf{P}^{H} \mathbf{P}-2 \operatorname{Re}\left(\mathbf{P}^{H} \mathbf{A D}\right)+\mathbf{D}^{H} \mathbf{A}^{H} \mathbf{A D}\right) \\
\text { subject to }\|\mathbf{D}\|_{F}^{2}=\beta .
\end{gathered}
$$

Using the orthogonality of columns of the matrix $\mathbf{A}$, that is, $\mathbf{A}^{H} \mathbf{A}=N_{t} \mathbf{I}$, the last term in the above objective is $\beta N_{t}$, which is a constant. Thus, after ignoring the constant terms in the objective of the above problem we get,

$$
\begin{gathered}
\mathcal{P}_{16}: \min _{\mathbf{D}}\left\|\mathbf{A}^{H} \mathbf{P}-\mathbf{D}\right\|_{F}^{2} \\
\text { subject to }\|\mathbf{D}\|_{F}^{2}=\beta .
\end{gathered}
$$

It is easy to see that the above problem is a projection problem for which the solution admits a closed-form given by,

$$
\mathbf{D}=\beta \frac{\mathbf{A}^{H} \mathbf{P}}{\left\|\mathbf{A}^{H} \mathbf{P}\right\|_{F}} .
$$

Substituting back this solution into the objective function of problem $\mathcal{P}_{12}$ we get,

$$
\begin{gathered}
\mathcal{P}_{17}: \min _{\mathbf{A}}\left\|\mathbf{P}-\beta \mathbf{A} \frac{\mathbf{A}^{H} \mathbf{P}}{\left\|\mathbf{A}^{H} \mathbf{P}\right\|_{F}}\right\|_{F}^{2} \\
\text { subject to } \mathbf{A} \in \mathcal{C} .
\end{gathered}
$$

The objective function of $\mathcal{P}_{17}$ is simplified after expanding it as,

$$
\begin{gathered}
\operatorname{Tr}\left\{\mathbf{P}^{H} \mathbf{P}-2 \beta \operatorname{Re}\left(\mathbf{P}^{H} \mathbf{A} \frac{\mathbf{A}^{H} \mathbf{P}}{\left\|\mathbf{A}^{H} \mathbf{P}\right\|_{F}}\right)\right\} \\
+\beta^{2} \operatorname{Tr}\left\{\frac{\mathbf{P}^{H} \mathbf{A}}{\left\|\mathbf{A}^{H} \mathbf{P}\right\|_{F}} \mathbf{A}^{H} \mathbf{A} \frac{\mathbf{A}^{H} \mathbf{P}}{\left\|\mathbf{A}^{H} \mathbf{P}\right\|_{F}}\right\} .
\end{gathered}
$$

Further simplification leads to the following function,

$$
-\beta\left\|\mathbf{A}^{H} \mathbf{P}\right\|_{F}+\beta^{2} N_{t}
$$

Thus, after ignoring the constant terms, we have the following problem,

$$
\begin{gathered}
\mathcal{P}_{18}: \min _{\mathbf{A}}-\left\|\mathbf{A}^{H} \mathbf{P}\right\|_{F} \\
\text { subject to } \mathbf{A} \in \mathcal{C} .
\end{gathered}
$$

Equivalently, the above problem can be reformulated as the following maximization problem,

$$
\begin{gathered}
\mathcal{P}_{19}: \max _{\mathbf{A}}\left\|\mathbf{A}^{H} \mathbf{P}\right\|_{F}^{2} \\
\text { subject to } \mathbf{A} \in \mathcal{C} .
\end{gathered}
$$

It is easy to see that the above objective can be rewritten as,

$$
\begin{gathered}
\mathcal{P}_{20}: \max _{\mathbf{A}} g(\mathbf{A})=\sum_{i=1}^{N_{t}} \tilde{\mathbf{a}}_{i}^{H} \mathbf{P} \mathbf{P}^{H} \tilde{\mathbf{a}}_{i} \\
\text { subject to } \mathbf{A} \in \mathcal{C},
\end{gathered}
$$

where $\tilde{\mathbf{a}}_{i}$ is the $i$-th column of the matrix $\mathbf{A}$. It is important to note that the objective function in $\mathcal{P}_{20}$ is a sum of $N_{t}$ quadratic convex functions. It is a well-known fact that each quadratic convex function is lower bounded by its first-order Taylor's expansion [45]. Therefore, adopting the minorizationmaximization (MM) framework, we first construct a surrogate function using the first-order Taylor's expansion which is a tight minorizer of the original function. Using this fact each function of the sum of the objective function in $\mathcal{P}_{20}$ is minorized as,

$$
\begin{array}{rl}
\tilde{\mathbf{a}}_{i}^{H} \mathbf{P} \mathbf{P}^{H} \tilde{\mathbf{a}}_{i} \geq \tilde{\mathbf{a}}_{i, k}^{H} & \mathbf{P} \mathbf{P}^{H} \tilde{\mathbf{a}}_{i, k} \\
& +2 \operatorname{Re}\left(\left(\tilde{\mathbf{a}}_{i}-\tilde{\mathbf{a}}_{i, k}\right)^{H} \mathbf{P} \mathbf{P}^{H} \tilde{\mathbf{a}}_{i, k}\right) .
\end{array}
$$

Thus, the overall objective can be minorized as,

$$
\begin{aligned}
g(\mathbf{A}) \geq \tilde{g}\left(\mathbf{A} ; \mathbf{A}_{k}\right) & =\sum_{i=1}^{N_{t}} \tilde{\mathbf{a}}_{i, k}^{H} \mathbf{P} \mathbf{P}^{H} \tilde{\mathbf{a}}_{i, k} \\
& +2 \operatorname{Re}\left(\left(\tilde{\mathbf{a}}_{i}-\tilde{\mathbf{a}}_{i, k}\right)^{H} \mathbf{P} \mathbf{P}^{H} \tilde{\mathbf{a}}_{i, k}\right)
\end{aligned}
$$

Similar to the properties satisfied by a valid majorizer from (22)-(24), the above surrogate function also satisfies the same properties except with reversed inequality in (22), since it minorizes the original function. Therefore, after neglecting the constant terms of the above surrogate function, the minorized version of the problem $\mathcal{P}_{20}$ can be written as,

$$
\begin{gathered}
\mathcal{P}_{21}: \max _{\mathbf{A}} \sum_{i=1}^{N_{t}} \operatorname{Re}\left(\tilde{\mathbf{a}}_{i}^{H} \mathbf{P} \mathbf{P}^{H} \tilde{\mathbf{a}}_{i, k}\right) \\
\text { subject to } \mathbf{A} \in \mathcal{C} .
\end{gathered}
$$

Problem $\mathcal{P}_{21}$ can be further reformulated to,

$$
\begin{gathered}
\mathcal{P}_{22}: \min _{\mathbf{A}} \sum_{i=1}^{N_{t}}\left\|\tilde{\mathbf{a}}_{i}-\mathbf{P} \mathbf{P}^{H} \tilde{\mathbf{a}}_{i, k}\right\|_{2}^{2} \\
\text { subject to } \mathbf{A} \in \mathcal{C} .
\end{gathered}
$$

The latter formulation is derived by using the fact that each block of the matrix is a vector having only $T_{x} / N_{t}$ non-zero 


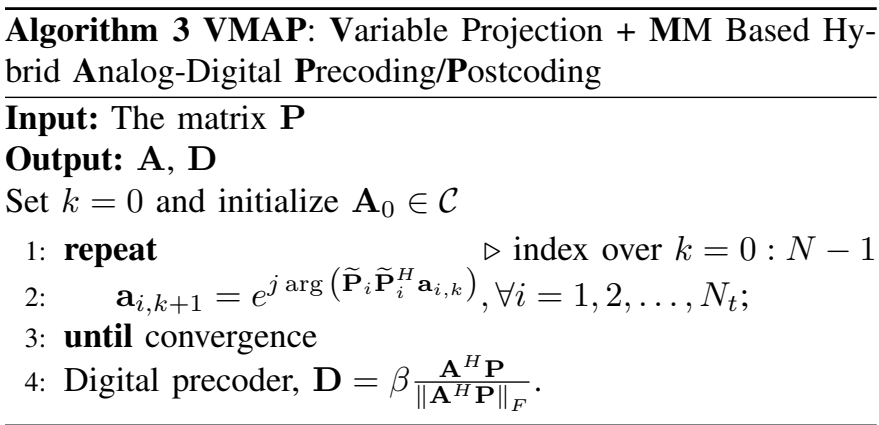

elements according to the structure presented in (1). It means, that only a sub-matrix of $\mathbf{P} \mathbf{P}^{H}$ is required to get the next update. Thus, we arrive at the following formulation of the problem,

$$
\begin{aligned}
\mathcal{P}_{23}: & \min _{\mathbf{A}} \\
\text { subject to } \mathbf{A} \in \mathcal{C}, & \sum_{i=1}^{N_{t}}\left\|\mathbf{a}_{i}-\widetilde{\mathbf{P}}_{i} \widetilde{\mathbf{P}}_{i}^{H} \mathbf{a}_{i, k}\right\|_{2}^{2}
\end{aligned}
$$

where $\mathbf{a}_{i}$ is the $i$-th non-zero block of matrix $\mathbf{A}$ as shown in (1) and $\mathbf{a}_{i, k}$ is its corresponding $k$-th iterate. The matrix $\widetilde{\mathbf{P}}_{i}=\mathbf{P}_{i: i+\frac{T x}{N_{t}}-1,:}$ is the $i$-th submatrix formed by extracting the $i$-th to $\left(i+\frac{T_{x}}{N_{t}}-1\right)$-th rows of matrix $\mathbf{P}$. Therefore, the problem can be solved in a parallel manner for all $N_{t}$ blocks simultaneously. It is readily seen that problem $\mathcal{P}_{23}$ is a least-squares projection problem and admits the following closed-form solution,

$$
\mathbf{a}_{i}=e^{j \arg \left(\widetilde{\mathbf{P}} \widetilde{\mathbf{P}}^{H} \mathbf{a}_{i, k}\right)}, \forall i=1,2, \ldots, N_{t} .
$$

Thus, by using the minorization-maximization framework, once we converge for the analog precoding, the solution of the digital BB precoding matrix is given by (35). The complete algorithm to design the hybrid precoder from the fully digital precoder, henceforth referred to as VMAP (Variable Projection + MM Based Hybrid Analog- Digital Precoding/Postcoding), is presented in Algorithm 3.

\section{B. Convergence Analysis of Algorithm 3}

We now, prove the convergence of the iterates generated by the algorithm VMAP to the set of stationary points. Since this framework first eliminates the variable $\mathbf{D}$, we only need to show the convergence of the sequence $\left\{\mathbf{A}_{k}\right\}$.

Theorem IV.1. Let $\left\{\mathbf{A}_{k}\right\}$ be the sequence of iterates generated by Algorithm VMAP. Then, every limit point of the sequence $\left\{\mathbf{A}_{k}\right\}$ is a stationary point of problem $\mathcal{P}_{19}$.

Proof. See Appendix B.

\section{Complexity of Algorithm VMAP}

Algorithm VMAP requires a matrix-matrix, matrix-vector multiplications and phase computation operation at each iteration. As compared to SDR-ALTMIN proposed in [12], here we do not require to solve the problem using alternating minimization. The SDR-ALTMIN algorithm solves a nonconvex QCQP using semidefinite relaxation and uses solvers

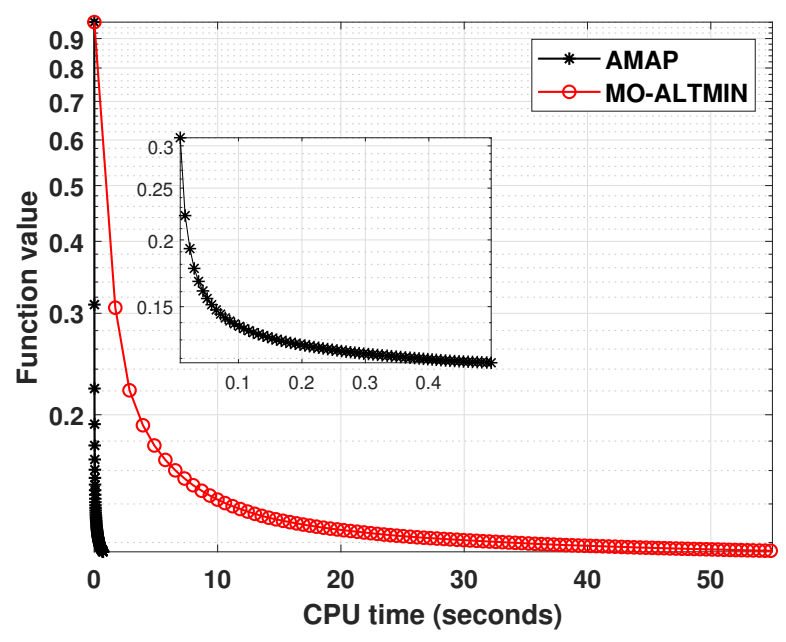

Fig. 1. Objective function variation with time for the fully connected architecutre, $N_{t}=N_{r}=N_{s}=6$.

like CVX, SeduMi to solve the problem. As compared to this, our algorithm does not require any external solvers, thus more computationally efficient. Similar to the fully connected architecture the worst-case complexity to carry out matrix multiplication of the term $\widetilde{\mathbf{P}}_{i} \widetilde{\mathbf{P}}_{i}^{H}$ is $O\left(\left(T_{x} / N_{t}\right)^{2} N_{s}\right)$. The exponential operation can be carried in $O\left(\left(T_{x} / N_{t}\right) N_{s}\right)$ operations. Thus, our algorithm is much more computationally efficient and is easily scalable to large dimensions.

\section{Simulation Results}

In this section, we provide numerical simulation results to test the performance of proposed algorithms and show their potential under different regimes. In the considered scenario a transmitter of $T_{x}=144$ and a receiver of $R_{x}=36$ is assumed. The channel parameters are $N_{c}=5$ clusters and $N_{p}=10$ propagation paths. The element spacing of the ULA is set to $d=\frac{\lambda}{2}$. Next, we study the evolution of the objective function with the number of iterations and the CPU time required to converge for the AMAP and MO-ALTMIN algorithms.

\section{A. Objective Value Comparison}

First, we compare the evolution of the objective function of the problem $\mathcal{P}_{1}$ with CPU time for the fully-connected case. As a benchmark, we compare the performance proposed algorithm AMAP with MO-ALTMIN algorithm from [12]. The comparison is made in terms of the CPU time required by the two algorithms to converge with a fixed number of iterations. The running time for the algorithms consists of the computation of the two precoding matrices. As both algorithms work on the philosophy of alternating minimization, we initialize them with the same analog precoding matrix. It is readily seen from Figures 1 and 2 that the AMAP algorithm and the MO-ALTMIN algorithm perform very close to each other, though AMAP converges much faster. Note that we have not shown other existing codebook free approaches from [24], [25] as they optimize different objective functions. But later, we do compare them on the basis of the SE achieved. 


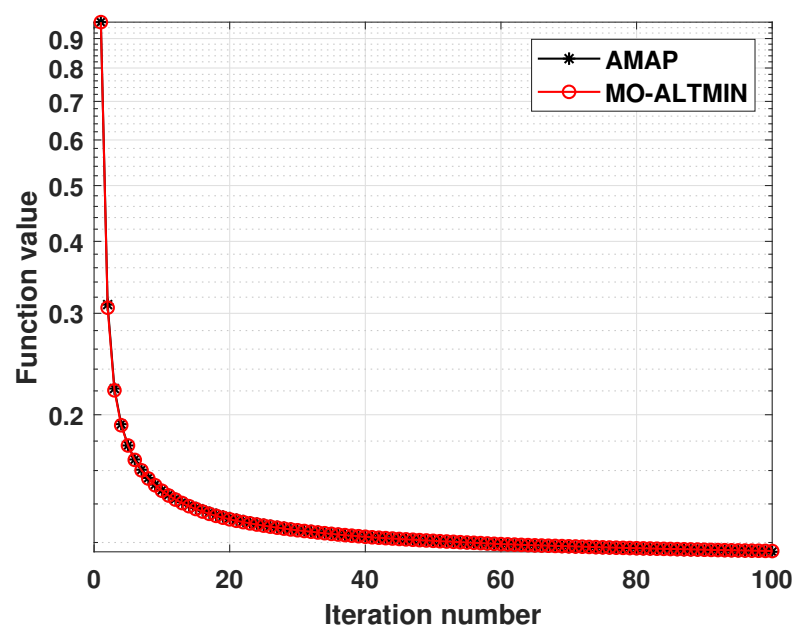

Fig. 2. Objective function variation with iteration for the fully connected architecutre, $N_{t}=N_{r}=N_{s}=6$.

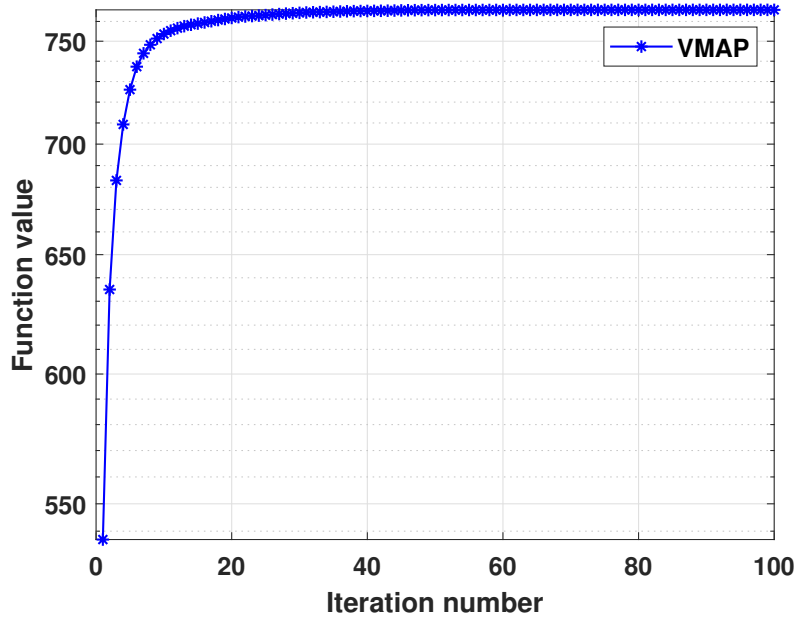

Fig. 3. Objective function variation with the number of iterations for the partially connected architecture, $N_{t}=N_{r}=N_{s}=6$.

For the partially connected architecture, the objective function evolution of problem $\mathcal{P}_{21}$ with CPU time is shown in Figure 3. As expected, the objective function value monotonically increases with the number of iterations and converges.

\section{B. Spectral Efficiency Comparison}

In order to compute the spectral efficiency of the system, we use the same algorithms at the receiver's side. As already mentioned, both the problems at the transmitter's as well as at the receiver's have the same mathematical structure, with a change in the dimensions at the two ends. For the fully connected case, the spectral efficiency achieved by different algorithms when $N_{t}=N r=N_{s}=6$, is shown in Figure 4 . As it is readily seen from there apart from MO-ALTMIN, we have compared the results with other existing works including OMP [3], phase projection [24], Alt-MaG [25] algorithms and the fully digital precoder designed by the singular value

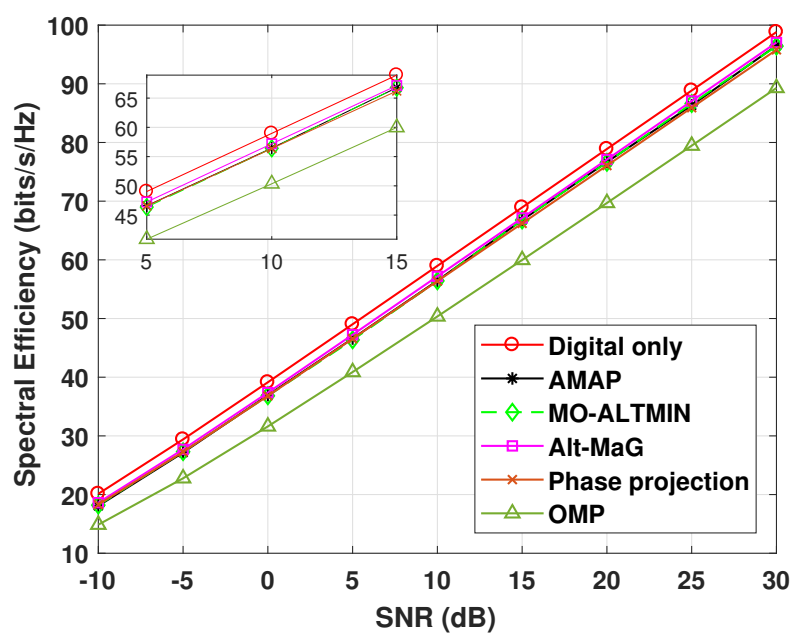

Fig. 4. Spectral efficiency comparison for different algorithms for the fully connected architecture, $N_{t}=N_{r}=N_{s}=6$.

decomposition (SVD) of the channel matrix and the waterfilling algorithm [39]. The hybrid A/D precoder and postcoder updates for the Alt-MaG algorithm are obtained using the MaGiQ algorithm proposed in the same referenced work. The primary reason behind showing the SE achieved by a fully digital precoder is to compare the loss in SE achieved by employing the hybrid architecture. The proposed algorithm achieve the SE efficiency closed to the one achieved by the fully digital precoder. It is also observed that all the algorithms perform very close to the fully-digital case, whereas Figure 1 reveals that AMAP algorithm gains significantly on the time required to converge in comparison with the MO-ALTMIN algorithm.

Next, we consider the SE variation with the different number of RF chains for the fully connected architecture, by fixing the number of streams. We consider the variation only in the case when $N_{s} \leq N_{t}<2 N_{s}$; this is because when the number of RF chains becomes twice the number of streams, the SE achieved by fully connected architecture coincides with the system employing fully digital precoding. The variation with the $N_{t}$ is shown in Figure 5. Once again, we perform similar to the MO-ALTMIN algorithm, whereas the phase projection based algorithm [24] and Alt-MaG from [25] does not scale well with the number of RF chains.

The SE efficiency comparison for the partially connected architecture is shown in Figure 6. It is readily observed that the SE achieved by our algorithm is significantly larger than to that of SDR-ALTMIN algorithm.

The SE efficiency variation with the number of RF chains is shown in Figure 7. To get the block-diagonal structure and to avoid divisibility issues in constructing the analog precoding matrices, we have used a different arrangement with $T_{x}=144$ and $R_{x}=72$, such that $T_{x} / N_{t}$ and correspondingly $R_{x} / N_{r}$ are integers. It is clear from the results that the proposed VMAP algorithm provides improved performance compared to the SDR-ALTMIN algorithm. 


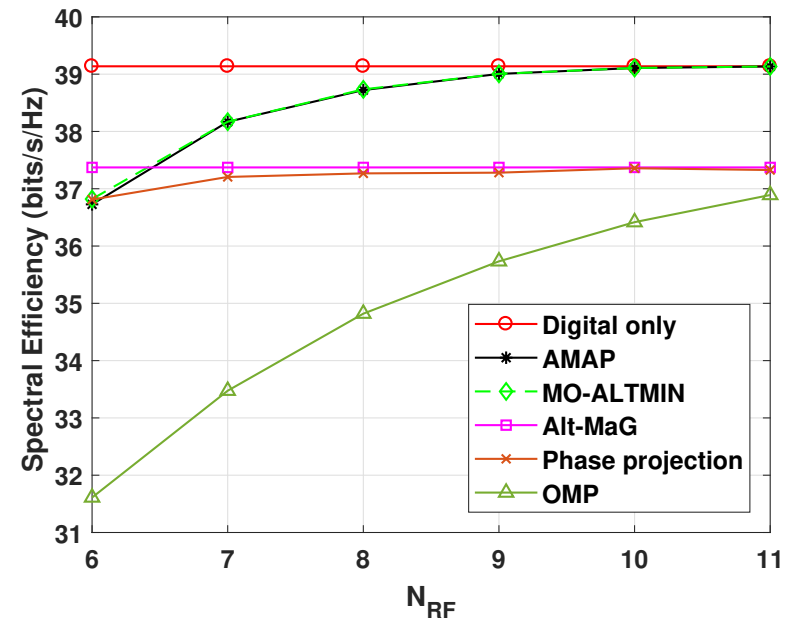

Fig. 5. Spectral Efficiency comparison for different algorithm for the fully connected architecture, when $N_{s}=6, N_{t}=N_{r}=N_{\mathrm{RF}}$, and SNR $=0 \mathrm{~dB}$.

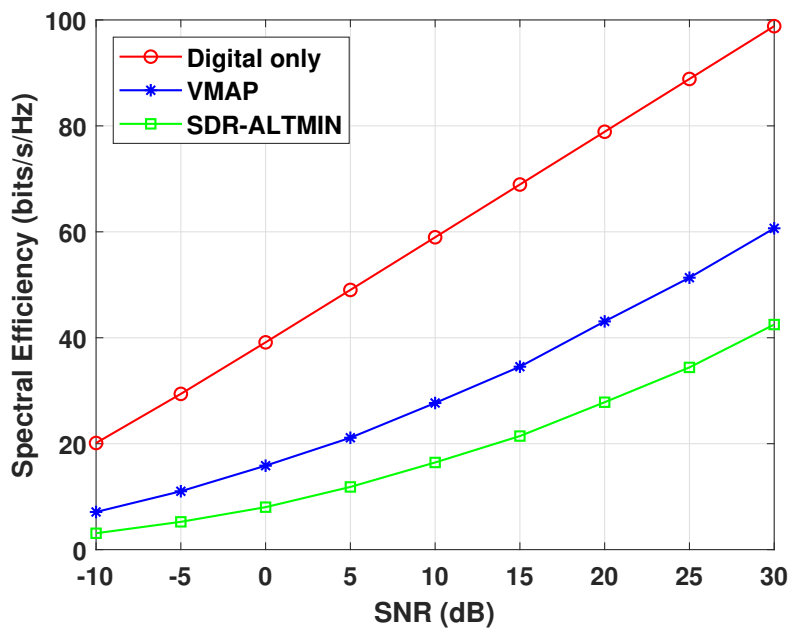

Fig. 6. Spectral Efficiency comparison for different algorithm for the partially connected architecture, when $N_{t}=N_{r}=N_{s}=6$.

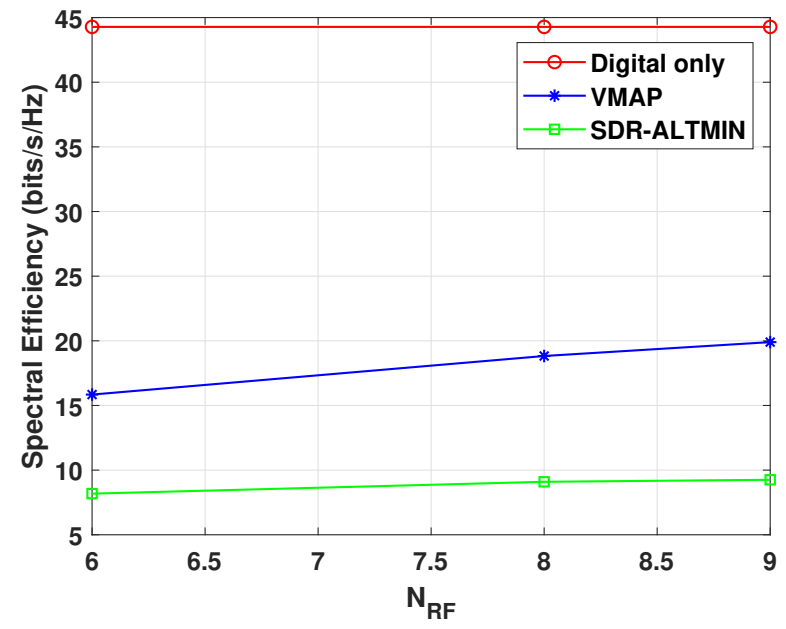

Fig. 7. Spectral Efficiency comparison for different algorithm for the partially connected architecture, when $N_{s}=6, N_{t}=N_{r}=N_{\mathrm{RF}}$, and SNR $=0 \mathrm{~dB}$

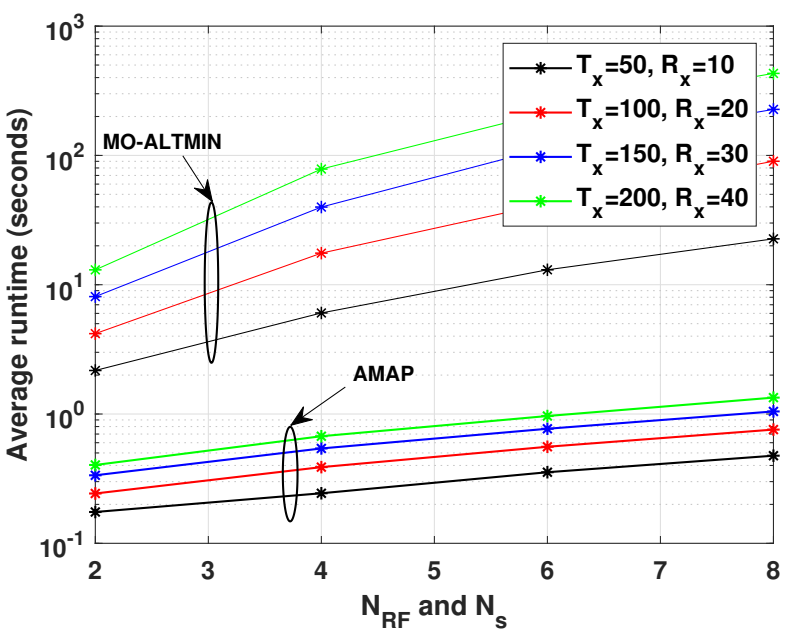

Fig. 8. Average running times variation with the number of RF chains and the number of streams under different system dimensions for the fully connected architecutre, $\mathrm{SNR}=0 \mathrm{~dB}$.

\section{Scalability of the Algorithms}

In this section, we present simulation results to investigate the scalability of the proposed algorithms. As AMAP and MOALTMIN algorithms minimize the same objective we compare their average running time. For the remaining algorithms, later we provide a table summarizing the average time required to design the complete transceiver as they consider different optimization problems. We plot in Figure 8, the average running time for the AMAP and MO-ALTMIN algorithm with the number of streams as well as with the different number of transmitting and receiving antennas.

We increase both the number of streams and RF chains for each arrangement having a different number of transmitting and receiving antennas. It is easy to see that the proposed algorithms are much faster than the MO-ALTMIN algorithm. Table I shows the comparison of SE achieved by the different techniques along with the time required to design the fullyconnected hybrid transceiver at a SNR of $0 \mathrm{~dB}$. There, $\mathrm{SE}_{X}$ represents the $\mathrm{SE}$ achieved in bits/s/Hz and $\mathrm{T}_{\mathrm{X}}$ denotes the time required in seconds, with the subscript $\mathrm{X}$ representing the names of different techniques. In the referred table, we have fixed the same number of maximum iterations for the AMAP and Alt-MaG algorithms. It is observed that the proposed algorithms achieve better SE in comparison with the other algorithms, with significant gains in the average run time. The phase projection has lower computational complexity among all the algorithms but it has marginal improvements in the $\mathrm{SE}$ achieved with the number of RF chains. Therefore, the proposed algorithm AMAP is a good compromise between the complexity and the performance.

A comparison of the VMAP and SDR-ALTMIN algorithms for the partially connected case is shown in Figure 9. It is observed that the VMAP algorithm has many orders lower average running time than the SDR-ALTMIN algorithm. 
TABLE I

AVERAGE RUNNING TIME AND SE ACHIEVED BY DIFFERENT ALGORITHMS, $T_{x}=64, R_{x}=16$ AND $L_{s}=6$

\begin{tabular}{|c||c|c|c|c|c|c|c|c|}
\hline \multicolumn{8}{|c|}{ Average running time (in seconds) and SE (in bits/s/Hz) achieved } \\
\hline $\begin{array}{c}N_{t} \text { and } N_{r}= \\
N_{\mathrm{RF}}\end{array}$ & $\mathrm{T}_{\text {AMAP }}$ & $\mathrm{SE}_{\text {AMAP }}$ & $\mathrm{T}_{\text {MO-ALTMIN }}$ & $\mathrm{SE}_{\text {MO-ALTMIN }}$ & $\mathrm{T}_{\text {Alt-MaG }}$ & $\mathrm{SE}_{\text {Alt-MaG }}$ & $\mathrm{T}_{\text {Phase projection }}$ & SE Phase projection \\
\hline 6 & 0.8688 & 24.3515 & 16.1905 & 24.3996 & 4.2433 & 24.7777 & 0.1771 & 24.3336 \\
\hline 7 & 0.9689 & 25.4934 & 21.8647 & 25.5188 & 4.2696 & 24.7777 & 0.2042 & 24.4707 \\
\hline 8 & 1.0430 & 25.8546 & 26.2132 & 25.8553 & 4.1964 & 24.7777 & 0.2228 & 24.5241 \\
\hline 9 & 1.2021 & 25.9699 & 32.0642 & 25.9696 & 4.3754 & 24.7777 & 0.2660 & 24.6410 \\
\hline 10 & 1.2665 & 26.0067 & 37.6997 & 26.0068 & 4.1591 & 24.7777 & 0.2841 & 24.6967 \\
\hline 11 & 1.4505 & 26.0121 & 50.1005 & 26.0121 & 4.4254 & 24.7777 & 0.3526 & 24.6388 \\
\hline
\end{tabular}

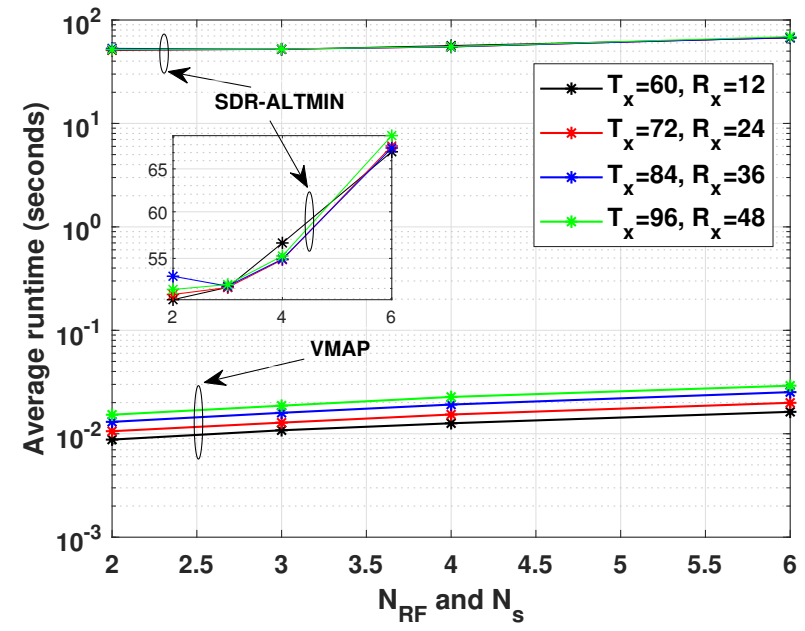

Fig. 9. Average running times variation with the number of RF chains and the number of streams under different system dimensions for the partially connected architecutre, $\mathrm{SNR}=0 \mathrm{~dB}$.

\section{CONCLUSIONS}

In this paper, we have studied the problem of hybrid analogdigital transceiver design for a mmWave MIMO system. We have proposed two algorithms to solve the hybrid design problem for the fully connected and the partially connected architectures, respectively. For the fully connected case, the AMAP algorithm was developed based on the majorizationminimization and the alternating minimization frameworks. Convergence to a stationary point of the iterates generated from the algorithm was also established. An efficient algorithm namely, VMAP was developed for the partially connected case. First, the digital BB precoding matrix was eliminated from the objective function and the problem for analog precoding was solved using the minorization-maximization framework. Once, the algorithm converges to a stationary point, the digital precoder then obtained in closed-form. From the numerical simulation results, we list the following observations which unveil the importance of our algorithms:

- The AMAP algorithm converged faster compared to the state-of-the-art solution in literature. The hybrid precoding matrices obtained from the proposed algorithm had similar performance to the state-of-the-art MO-ALTMIN algorithm in terms of SE of the system.

- The VMAP algorithm easily scaled to larger system dimensions and significantly gained on the SE achieved as compared to the SDR-ALTMIN algorithm.

- Both the proposed algorithms scaled to larger system dimensions with several orders of gain on average running time.

Our results clearly demonstrate the aforementioned gains of the proposed algorithms by utilizing a new approach based on the MM framework, variable elimination and the alternating minimization with provable convergence guarantees.

\section{APPENDIX A}

PROOF OF THEOREM III.3

Proof. To show the convergence of the iterates to a KarushKuhn-Tucker (KKT) point, we start with the properties of the surrogate function from (22)-(24) for the analog precoding problem and show that the iterates obtained decreases the objective function monotonically. To show that, we first write the updates of the algorithm AMAP as,

$$
\begin{aligned}
& \mathbf{A}_{k+1}=\arg \min _{\mathbf{A} \in \mathcal{A}} \tilde{f}\left(\mathbf{A} ; \mathbf{A}_{k}, \mathbf{D}_{k}\right) \\
& \mathbf{D}_{k+1}=\arg \min _{\mathbf{D}} f\left(\mathbf{A}_{k+1}, \mathbf{D}\right),
\end{aligned}
$$

where the solution $\mathbf{A}_{k}$ for the matrix $\mathbf{A}$ is obtained after the convergence of Algorithm 1 convergence, $k$ represents the iteration number of the AMAP algorithm and the update for matrix $\mathbf{D}$ is obtained from the closed form solution. From property (23) we have,

$$
\begin{aligned}
f\left(\mathbf{A}_{k}, \mathbf{D}_{k}\right) & =\tilde{f}\left(\mathbf{A}_{k} ; \mathbf{A}_{k}, \mathbf{D}_{k}\right), \\
& \geq \tilde{f}\left(\mathbf{A}_{k+1} ; \mathbf{A}_{k}, \mathbf{D}_{k}\right), \\
& \geq f\left(\mathbf{A}_{k+1}, \mathbf{D}_{k}\right), \\
& \geq f\left(\mathbf{A}_{k+1}, \mathbf{D}_{k+1}\right),
\end{aligned}
$$

where $\mathbf{A}_{k}$ and $\mathbf{A}_{k+1}$ are the minimizers obtained from Algorithm 1. Equation (50) follows from the descent property of the MM framework [40], which is $f\left(\mathbf{A}_{l+1}, \mathbf{D}_{k}\right) \leq$ $\tilde{f}\left(\mathbf{A}_{l+1} ; \mathbf{A}_{k+1}, \mathbf{D}_{k}\right) \leq \tilde{f}\left(\mathbf{A}_{l} ; \mathbf{A}_{k}, \mathbf{D}_{k}\right) \leq f\left(\mathbf{A}_{l}, \mathbf{D}_{k}\right)$. The inequality (51) holds because of (22), and (52) follows from (48). Therefore, $\left\{f\left(\mathbf{A}_{k}, \mathbf{D}_{k}\right)\right\}$ is a monotonically decreasing sequence and it thus converges, since it is lower bounded. Consider a convergent subsequence $\left\{\mathbf{A}_{s_{k}}, \mathbf{D}_{s_{k}}\right\}$, indexed by 
$s_{k}$, converging to the limit-point $\left\{\mathbf{A}_{\infty}, \mathbf{D}_{\infty}\right\}$. Then, we can write,

$$
\begin{aligned}
\tilde{f}\left(\mathbf{A} ; \mathbf{A}_{s_{k}}, \mathbf{D}_{s_{k}}\right) & \geq \tilde{f}\left(\mathbf{A}_{s_{k}+1} ; \mathbf{A}_{s_{k}}, \mathbf{D}_{s_{k}}\right) \\
& \geq f\left(\mathbf{A}_{s_{k}+1}, \mathbf{D}_{s_{k}}\right) \\
& \geq f\left(\mathbf{A}_{s_{k}+1}, \mathbf{D}_{s_{k}+1}\right) \\
& \geq f\left(\mathbf{A}_{s_{k+1}}, \mathbf{D}_{s_{k+1}}\right) \\
& =\tilde{f}\left(\mathbf{A}_{s_{k+1}} ; \mathbf{A}_{s_{k+1}}, \mathbf{D}_{s_{k+1}}\right) .
\end{aligned}
$$

The inequality (53) follows from (47), (54) holds because of (22), (55) follows from (48), (56) comes from (48), and equation (57) is obtained from (23). Since, $\tilde{f}\left(\mathbf{A}_{s_{k+1}} ; \mathbf{A}_{\mathbf{s}_{\mathbf{k}+1}}, \mathbf{D}_{s_{k+1}}\right)$ is a continuous function, we can take the following limit,

$$
\begin{gathered}
\lim _{k \rightarrow \infty} \tilde{f}\left(\mathbf{A} ; \mathbf{A}_{s_{k}}, \mathbf{D}_{s_{k}}\right) \geq \lim _{k \rightarrow \infty} \tilde{f}\left(\mathbf{A}_{s_{k+1}} ; \mathbf{A}_{s_{k+1}}, \mathbf{D}_{s_{k+1}}\right) \\
\tilde{f}\left(\mathbf{A} ; \mathbf{A}_{\infty}, \mathbf{D}_{\infty}\right) \geq \tilde{f}\left(\mathbf{A}_{\infty} ; \mathbf{A}_{\infty}, \mathbf{D}_{\infty}\right), \forall \mathbf{A} \in \mathcal{A},
\end{gathered}
$$

which means that $\mathbf{A}_{\infty}$ is a coordinate-wise minimizer of $\tilde{f}\left(\mathbf{A} ; \mathbf{A}_{\infty}, \mathbf{D}_{\infty}\right)$, and thus, it satisfies the partial KKT condition with respect to $\mathbf{A}$, that is,

$$
\begin{array}{r}
\nabla_{\mathbf{A}} \tilde{f}\left(\mathbf{A}_{\infty} ; \mathbf{A}_{\infty}, \mathbf{D}_{\infty}\right)+2 \mathbf{\Psi}_{1} \circ \mathbf{A}_{\infty}=\mathbf{0} \\
\nabla_{\mathbf{A}} f\left(\mathbf{A}_{\infty}, \mathbf{D}_{\infty}\right)+2 \Psi_{1} \circ \mathbf{A}_{\infty}=\mathbf{0}
\end{array}
$$

where $\Psi_{1}$ is the dual variable associated with the squared unit-modulus constraint. The last equation holds due to the gradient-consistency between the surrogate and the original function, as depicted in (24). Working on the similar lines and using (48), we derive the following,

$$
f\left(\mathbf{A}_{s_{k}}, \mathbf{D}\right) \geq f\left(\mathbf{A}_{s_{k}}, \mathbf{D}_{s_{k}}\right) .
$$

By similar arguments made earlier, we can take the limit $k \rightarrow$ $\infty$ on both sides of the above inequality, resulting in,

$$
f\left(\mathbf{A}_{\infty}, \mathbf{D}\right) \geq f\left(\mathbf{A}_{\infty}, \mathbf{D}_{\infty}\right) .
$$

This proves that $\mathbf{D}_{\infty}$ is coordinate-wise minimizer of $f\left(\mathbf{A}_{s_{k}}, \mathbf{D}\right)$ and thus, it satisfies the KKT condition for $\mathbf{D}$,

$$
\nabla_{\mathbf{D}} f\left(\mathbf{A}_{\infty}, \mathbf{D}_{\infty}\right)=\mathbf{0} .
$$

Therefore, using (59) and (62), it is readily established that the every limit point of the solution sequence is a KKT point. In order to show that the whole sequence $\left\{\mathbf{A}_{k}, \mathbf{D}_{k}\right\}$ together converges to the KKT point, we first establish the boundedness of the iterates. The solution obtained from Algorithm 1 lies in a compact set, and it is a well known result that if a set is compact then, it is bounded [46]. Another way to prove that a sequence is bounded easily follows from generalization of the boundedness of a vector sequence [42], [46]. A sequence $\left\{\mathbf{A}_{k}\right\}$ is bounded if and only if there exists a scalar $c$ such that $\left\|\mathbf{A}_{k}\right\|_{F} \leq c$, for all $k$ [42], [46]. Since, each entry of the matrix $\mathbf{A}_{k}$ is unit-modulus, it follows that $\left\|\mathbf{A}_{k}\right\|_{F} \leq \sqrt{T_{x} N_{t}}$. To prove that the sequence $\left\{\mathbf{D}_{k}\right\}$ is bounded, we use the similar argument and we have,

$$
\begin{aligned}
\|\mathbf{D}\|_{F} & \leq\left\|\left(\mathbf{A}^{H} \mathbf{A}\right)^{-1}\right\|_{2}\left\|\mathbf{A}^{H} \mathbf{P}\right\|_{F} \\
& \leq\left\|\left(\mathbf{A}^{H} \mathbf{A}\right)^{-1}\right\|_{2}\left\|\mathbf{A}^{H}\right\|_{F}\|\mathbf{P}\|_{F},
\end{aligned}
$$

where inequality (63) follows from $\|\mathbf{X Y}\|_{F} \leq\|\mathbf{X}\|_{2}\|\mathbf{Y}\|_{F}$ [47], $\|\mathbf{X}\|_{2}$ represents the spectral norm of the matrix $\mathbf{X}$ which is defined as the square-root of the maximum eigenvalue of matrix $\mathbf{X}^{H} \mathbf{X}$, that is $\sqrt{\lambda_{\max }\left(\mathbf{X}^{H} \mathbf{X}\right)}$. The last inequality (64) follows from Cauchy-Schwarz inequalty [47]. All the terms on right hand side of (64) are bounded, the spectral norm can be shown to be bounded if $\mathbf{A}$ is a full-rank matrix,

$$
\begin{aligned}
\left\|\left(\mathbf{A}^{H} \mathbf{A}\right)^{-1}\right\|_{2} & =\sqrt{\lambda_{\max }\left(\left(\mathbf{A}^{H} \mathbf{A}\right)^{-2}\right)} \\
& =\frac{1}{\lambda_{\min }\left(\mathbf{A}^{H} \mathbf{A}\right)} .
\end{aligned}
$$

From simulations it is observed that the matrix $\mathbf{A}$ is always full-column rank, thus $\left(\mathbf{A}^{H} \mathbf{A}\right)^{-1}$ exists. Hence, the quantity $\|\mathbf{D}\|_{F}<\infty$. Therefore, all the quantities on the right hand side of the inequality (64) are bounded, thus the sequence is bounded.

Now, consider that whole sequence does not converge to the set of KKT points $\mathcal{K}$. Therefore, there exists a subsequence not converging to any element of $\mathcal{K}$. But we have already shown, the boundedness of $\left\{\mathbf{A}_{k}\right\},\left\{\mathbf{D}_{k}\right\}$, which implies that every subsequence has a limit point and every limit point is a KKT point. This contradicts our assumption that the sequence does not converge to the set of KKT points. Thus, the whole sequence converges to a KKT point.

\section{APPENDIX B}

\section{ProOF OF THEOREM IV.1}

Proof. The objective function $g(\mathbf{A})$ of problem $\mathcal{P}_{19}$ is minorized by the surrogate function $\tilde{g}\left(\mathbf{A} ; \mathbf{A}_{k}\right)$ from (42), where $\mathbf{A}_{k}$ is the feasible solution available at $k$-th iteration. According to the MM framework and the similar arguments presented in section III-D, we have,

$$
g\left(\mathbf{A}_{k+1}\right) \geq \tilde{g}\left(\mathbf{A}_{k+1} ; \mathbf{A}_{k}\right) \geq \tilde{g}\left(\mathbf{A}_{k} ; \mathbf{A}_{k}\right)=f\left(\mathbf{A}_{k}\right),
$$

this means that the sequence $\left\{g\left(\mathbf{A}_{k}\right)\right\}$ increases monotonically. Assume that there exists a subsequence $\left\{\mathbf{A}_{k_{j}}\right\}$ converging to $\mathbf{A}_{\infty}$, then we can write,

$$
\begin{aligned}
\tilde{g}\left(\mathbf{A}_{k_{j+1}} ; \mathbf{A}_{k_{j+1}}\right) & =g\left(\mathbf{A}_{k_{j+1}}\right) \geq f\left(\mathbf{A}_{k_{j}+1}\right) \\
& \geq \tilde{g}\left(\mathbf{A}_{k_{j}+1} ; \mathbf{A}_{k_{j}}\right) \geq \tilde{g}\left(\mathbf{A} ; \mathbf{A}_{k_{j}}\right), \forall \mathbf{A} \in \mathcal{C} .
\end{aligned}
$$

Now, using the continuity of the function $\tilde{f}(\cdot)$ and taking the limit as $j \rightarrow \infty$,

$$
\begin{aligned}
\tilde{g}\left(\lim _{j \rightarrow \infty} \mathbf{A}_{k_{j+1}}\right) & \geq \tilde{g}\left(\mathbf{A} ; \lim _{j \rightarrow \infty} \mathbf{A}_{k_{j}}\right) \\
\tilde{g}\left(\mathbf{A}_{\infty} ; \mathbf{A}_{\infty}\right) & \geq \tilde{g}\left(\mathbf{A} ; \mathbf{A}_{\infty}\right) .
\end{aligned}
$$

The above inequality means that $\mathbf{A}_{\infty}$ is a global maximizer of the function $\tilde{g}\left(\mathbf{A} ; \mathbf{A}_{\infty}\right)$ over the set $\mathcal{C}$. Thus, it satisfies the KKT condition,

$$
\nabla_{\mathbf{A}} \tilde{g}\left(\mathbf{A}_{\infty} ; \mathbf{A}_{\infty}\right)+2 \mathbf{\Psi}_{2} \circ \mathbf{A}_{\infty}=\mathbf{0} .
$$

where $\Psi_{2}$ is the dual variable associated with the squared constant modulus equality constraint. Since, $\mathbf{A}_{\infty}$ is a global maximizer of $\tilde{g}\left(\mathbf{A} ; \mathbf{A}_{\infty}\right)$, it should satisfy the first-order necessary condition as,

$$
\operatorname{Re}\left\{\operatorname{Tr}\left(\nabla_{\mathbf{A}} \tilde{g}\left(\mathbf{A}_{\infty} ; \mathbf{A}_{\infty}\right)^{H}\left(\mathbf{A}-\mathbf{A}_{\infty}\right)\right)\right\} \leq 0, \forall \mathbf{A} \in \mathcal{C}^{\prime}
$$


where $\mathcal{C}^{\prime}$ is the set constructed by replacing the squared constant modulus equality constraint of the set $\mathcal{C}$ with inequality. In other words, it is a convex approximation of the $\operatorname{set} \mathcal{C}$. Now, by consistency of the gradients between $g(\cdot)$, and $\tilde{g}(\cdot)$, we get,

$$
\begin{array}{cl}
\nabla_{\mathbf{A}} g\left(\mathbf{A}_{\infty}\right)+2 \mathbf{\Psi}_{2} \circ \mathbf{A}_{\infty} & =\mathbf{0}, \\
\operatorname{Re}\left\{\operatorname{Tr}\left(\nabla_{\mathbf{A}} g\left(\mathbf{A}_{\infty}\right)^{H}\left(\mathbf{A}-\mathbf{A}_{\infty}\right)\right)\right\} & \leq 0, \forall \mathbf{A} \in \mathcal{C}^{\prime} .
\end{array}
$$

The above inequality and the KKT condition implies that $\mathbf{A}_{\infty}$ is a stationary point of the problem $\mathcal{P}_{19}$.

\section{REFERENCES}

[1] X. Zhang, A. F. Molisch, and S. Y. Kung, "Variable-phase-shift-based RF-baseband codesign for MIMO antenna selection," IEEE Trans. Signal Process., vol. 53, no. 11, pp. 4091-4103, Nov 2005.

[2] A. Alkhateeb, O. El Ayach, G. Leus, and R. W. Heath, "Channel estimation and hybrid precoding for millimeter wave cellular systems," IEEE J. Sel. Topics Signal Process., vol. 8, no. 5, pp. 831-846, Oct 2014.

[3] O. E. Ayach, S. Rajagopal, S. Abu-Surra, Z. Pi, and R. W. Heath, "Spatially sparse precoding in millimeter wave MIMO systems," IEEE Trans. Wireless Commun., vol. 13, no. 3, pp. 1499-1513, March 2014.

[4] M. R. Akdeniz, Y. Liu, M. K. Samimi, S. Sun, S. Rangan, T. S Rappaport, and E. Erkip, "Millimeter wave channel modeling and cellular capacity evaluation," vol. 32, no. 6, pp. 1164-1179, June 2014.

[5] C. G. Tsinos, S. Maleki, S. Chatzinotas, and B. Ottersten, "On the energy-efficiency of hybrid analog-digital transceivers for single- and multi-carrier large Antenna array systems," IEEE J. Sel. Areas Commun. vol. 35, no. 9, pp. 1980-1995, Sep. 2017.

[6] D. J. Love and R. W. Heath, "Equal gain transmission in multiple-input multiple-output wireless systems," IEEE Trans. Commun., vol. 51, no. 7, pp. 1102-1110, July 2003.

[7] S. Hur, T. Kim, D. J. Love, J. V. Krogmeier, T. A. Thomas, and A. Ghosh, "Millimeter wave beamforming for wireless backhaul and access in small cell networks," IEEE Trans. Commun., vol. 61, no. 10, pp. 4391-4403, October 2013.

[8] T. Baykas, C. Sum, Z. Lan, J. Wang, M. A. Rahman, H. Harada, and S. Kato, "IEEE 802.15.3c: The first IEEE wireless standard for data rates over $1 \mathrm{~Gb} / \mathrm{s}, "$ IEEE Commun. Mag., vol. 49, no. 7, pp. 114-121, July 2011.

[9] A. F. Molisch, M. Z. Win, , and J. H. Winters, "Capacity of MIMO systems with antenna selection," IEEE Trans. Wireless Commun., vol. 4 no. 4, pp. 1759-1772, July 2005.

[10] A. Gorokhov, D. A. Gore, and A. J. Paulraj, "Receive antenna selection for MIMO spatial multiplexing: Theory and algorithms," IEEE Trans. Signal Process., vol. 51, no. 11, pp. 2796-2807, Nov 2003.

[11] S. Sanayei and A. Nosratinia, "Antenna selection in MIMO systems," IEEE Commun. Mag., vol. 42, no. 10, pp. 68-73, Oct 2004.

[12] X. Yu, J. Shen, J. Zhang, and K. B. Letaief, "Alternating minimization algorithms for hybrid precoding in millimeter wave MIMO systems," IEEE J. Sel. Topics Signal Process., vol. 10, no. 3, pp. 485-500, April 2016.

[13] T. E. Bogale, L. B. Le, A. Haghighat, and L. Vandendorpe, "On the number of RF chains and phase shifters, and scheduling design with hybrid analog-digital beamforming," IEEE Trans. Wireless Commun., vol. 15, no. 5, pp. 3311-3326, May 2016.

[14] S. Payami, M. Ghoraishi, and M. Dianati, "Hybrid beamforming for large antenna arrays with phase shifter selection," IEEE Trans. Wireless Commun., vol. 15, no. 11, pp. 7258-7271, Nov 2016.

[15] S. Payami, M. Ghoraishi, M. Dianati, and M. Sellathurai, "Hybrid beamforming with a reduced number of phase shifters for massive MIMO systems," IEEE Trans. Veh. Technol., vol. 67, no. 6, pp. 48434851, June 2018.

[16] A. Li and C. Masouros, "Hybrid analog-digital millimeter-wave MUMIMO transmission with virtual path selection," IEEE Commun. Lett., vol. 21, no. 2, pp. 438-441, Feb 2017.

[17] E. Vlachos, A. Kaushik, and J. Thompson, "Energy efficient transmitter with low resolution DACs for massive MIMO with partially connected hybrid architecture,' in 2018 IEEE 87th Vehicular Technology Conference (VTC Spring), June 2018, pp. 1-5.

[18] M. Á. Vázquez, L. Blanco, and A. I. Pérez-Neira, "Hybrid analog-digital transmit beamforming for spectrum sharing backhaul networks," IEEE Trans. Signal Process., vol. 66, no. 9, pp. 2273-2285, May 2018.
[19] C. G. Tsinos, S. Chatzinotas, and B. Ottersten, "Hybrid analog-digital transceiver designs for mmwave amplify-and-forward relaying systems," in 2018 41st International Conference on Telecommunications and Signal Processing (TSP), July 2018, pp. 1-6.

[20] C. G. Tsinos, S. Maleki, S. Chatzinotas, and B. Ottersten, "On the energy-efficiency of hybrid analog-digital transceivers for large antenna array systems," in 2017 IEEE International Conference on Communications (ICC), May 2017, pp. 1-7.

[21] — "Hybrid analog-digital transceiver designs for cognitive radio millimiter wave systems," in 2016 50th Asilomar Conference on Signals, Systems and Computers, Nov 2016, pp. 1785-1789.

[22] A. Liu and V. Lau, "Phase only RF precoding for massive MIMO systems With Limited RF Chains," IEEE Trans. Signal Process., vol. 62, no. 17 , pp. 4505-4515, Sep. 2014.

[23] X. Chen, A. Liu, Y. Cai, V. K. N. Lau, and M. Zhao, "Randomized two-timescale hybrid precoding for downlink multicell massive MIMO systems," IEEE Trans. Signal Process., vol. 67, no. 16, pp. 4152-4167, Aug 2019.

[24] C. Xing, X. Zhao, W. Xu, X. Dong, and G. Y. Li, "A framework on hybrid MIMO transceiver design based on matrix-monotonic optimization," IEEE Trans. Signal Process., vol. 67, no. 13, pp. 3531-3546, July 2019.

[25] S. S. Ioushua and Y. C. Eldar, "A family of hybrid analog-digital beamforming methods for massive MIMO systems," IEEE Trans. Signal Process., vol. 67, no. 12, pp. 3243-3257, June 2019.

[26] J. A. Tropp and A. C. Gilbert, "Signal recovery from random measurements via orthogonal matching pursuit," IEEE Trans. Inf. Theory, vol. 53, no. 12 , pp. 4655-4666, Dec 2007.

[27] J. A. Tropp, "Greed is good: Algorithmic results for sparse approximation," IEEE Trans. Inf. Theory, vol. 50, no. 10, pp. 2231-2242, Oct 2004.

[28] D. D. Lee and H. S. Seung, "Algorithms for non-negative matrix factorization," in Advances in Neural Information Processing Systems 13, T. K. Leen, T. G. Dietterich, and V. Tresp, Eds. MIT Press, 2001, pp. 556-562. [Online]. Available: http://papers.nips.cc/paper/1861algorithms-for-non-negative-matrix-factorization.pdf

[29] - "Learning the parts of objects by non-negative matrix factorization," Nature, vol. 401, no. 6755, p. 788, 1999.

[30] R. Bell, Y. Koren, and C. Volinsky, "Matrix factorization techniques for recommender systems," Computer, vol. 42, pp. 30-37, 082009. [Online]. Available: doi.ieeecomputersociety.org/10.1109/MC.2009.263

[31] P. Jain, P. Netrapalli, and S. Sanghavi, "Low-rank matrix completion using alternating minimization," in Proceedings of the Forty-fifth Annual ACM Symposium on Theory of Computing, ser. STOC '13. New York, NY, USA: ACM, 2013, pp. 665-674. [Online]. Available: http://doi.acm.org/10.1145/2488608.2488693

[32] E. J. Candés and B. Recht, "Exact matrix completion via convex optimization," Foundations of Computational Mathematics, vol. 9, no. 6 , p. 717, Apr 2009. [Online]. Available: https://doi.org/10.1007/s10208009-9045-5

[33] Y. Sun, P. Babu, and D. P. Palomar, "Majorization-minimization algorithms in signal processing, communications, and machine learning," IEEE Trans. Signal Process., vol. 65, no. 3, pp. 794-816, Feb 2017.

[34] J. Song, P. Babu, and D. P. Palomar, "Optimization methods for designing sequences with low autocorrelation sidelobes," IEEE Trans. Signal Process., vol. 63, no. 15, pp. 3998-4009, Aug 2015.

[35] A. F. Molisch, V. V. Ratnam, S. Han, Z. Li, S. L. H. Nguyen, L. Li, and K. Haneda, "Hybrid beamforming for massive MIMO: A survey," IEEE Commun. Mag., vol. 55, no. 9, pp. 134-141, Sep. 2017.

[36] P. F. M. Smulders and L. M. Correia, "Characterisation of propagation in $60 \mathrm{GHz}$ radio channels," Electronics Communication Engineering Journal, vol. 9, no. 2, pp. 73-80, April 1997.

[37] H. Xu, V. Kukshya, and T. S. Rappaport, "Spatial and temporal characteristics of $60-\mathrm{GHz}$ indoor channels," IEEE J. Sel. Areas Commun., vol. 20, no. 3, pp. 620-630, April 2002.

[38] D. P. Palomar and M. Chiang, "A tutorial on decomposition methods for network utility maximization," vol. 24, no. 8, pp. 1439-1451, Aug 2006.

[39] D. Tse and P. Viswanath, Fundamentals of Wireless Communication. Cambridge University Press, 2005.

[40] D. R. Hunter and K. Lange, "A tutorial on MM algorithms," The American Statistician, vol. 58, no. 1, pp. 30-37, 2004. [Online]. Available: https://doi.org/10.1198/0003130042836

[41] K. Lange, MM Optimization Algorithms. SIAM, 2016, vol. 147.

[42] D. P. Bertsekas, Nonlinear Programming. Taylor \& Francis, 1997, vol. 48 , no. 3 . 
[43] J. Tranter, N. D. Sidiropoulos, X. Fu, and A. Swami, "Fast unit-modulus least squares with applications in beamforming," IEEE Trans. Signal Process., vol. 65, no. 11, pp. 2875-2887, June 2017.

[44] G. Stewart, "A Krylov-Schur algorithm for large eigenproblems," SIAM Journal on Matrix Analysis and Applications, vol. 23, no. 3, pp. 601-614, 2002. [Online]. Available: https://doi.org/10.1137/S0895479800371529

[45] S. Boyd and L. Vandenberghe, Convex Optimization. Cambridge University Press, 2004.

[46] D. P. Bertsekas, A. Nedi, A. E. Ozdaglar et al., Convex Analysis and Optimization. Athena Scientific, 2003.

[47] G. H. Golub and C. F. Van Loan, Matrix Computations. JHU press, 2012, vol. 3

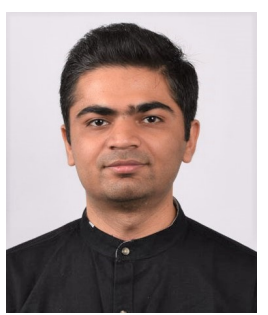

Aakash Arora (S‘18) received the M.S. (Research) degree in electrical engineering from the Indian Institute of Technology Delhi, India, in 2017. He is currently working towards the Ph.D. degree in computer science from the Interdisciplinary Centre for Security, Reliability and Trust, University of Luxembourg, Luxembourg. His research interests include optimization algorithms, statistical signal processing, wireless communications, and machine learning.

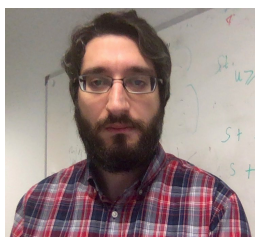

Christos G. Tsinos (S‘08-M`14) received the Diploma degree in computer engineering and informatics, the M.Sc. and Ph.D. degrees in signal processing and communication systems, and the M.Sc. degree in applied mathematics from the University of Patras, Greece, in 2006, 2008, 2013, and 2014, respectively. From 2014 to 2015, he was a PostDoctoral Researcher with the University of Patras. In 2015, he joined the Interdisciplinary Centre for Security, Reliability and Trust (SnT), University of Luxembourg, Luxembourg, as a ResearchAssociate. In the past, he was involved in a number of different $\mathrm{R} \& \mathrm{D}$ projects funded by national and/or EU funds. He is currently the PI of $\mathrm{R} \& \mathrm{D}$ Project ECLECTIC (Energy and CompLexity EffiCienT mIllimeter-wave Large-ArrayCommunications), funded under FNR CORE Framework. His current research interests include signal processing for mm-wave, massive MIMO, cognitive radio and satellite communications, and hyperspectral image processing. He is a member of the Technical Chamber of Greece.

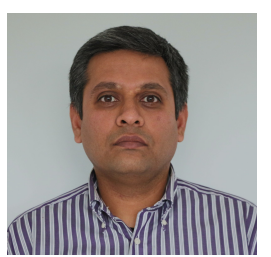

M. R. Bhavani Shankar received Masters and $\mathrm{Ph}$. D in Electrical Communication Engineering from Indian Institute of Science, Bangalore in 2000 and 2007 respectively. He was a Post Doc at the ACCESS Linnaeus Centre, Signal Processing Lab, Royal Institute of Technology (KTH), Sweden from 2007 to September 2009. He joined SnT in October 2009 as a Research Associate and is currently a Research Scientist at SnT. He was with Beceem Communications, Bangalore from 2006 to 2007 as a Staff Design Engineer working on Physical Layer algorithms for WiMAX compliant chipsets. He was a visiting student at the Communication Theory Group, ETH Zurich, headed by Prof. Helmut Blcskei during 2004. Prior to joining Ph. D, he worked on Audio Coding algorithms in Sasken Communications, Bangalore as a Design Engineer from 2000 to 2001. His research interests include Design and Optimization of MIMO Communication Systems, Automotive Radar and Array Processing, polynomial signal processing, Satellite communication systems, Resource Allocation, Game Theory and Fast Algorithms for Structured Matrices. He is currently on the Executive Committee of the IEEE Benelux joint chapter on communications and vehicular technology, member of the EURASIP Technical Area Committee on Theoretical and Methodological Trends in Signal Processing and serves as handling editor for Elsevier Signal Processing. He was a co-recipient of the 2014 Distinguished Contributions to Satellite Communications

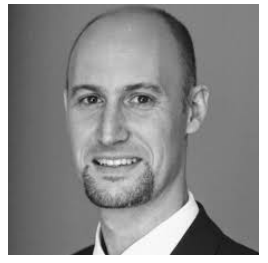

Symeon Chatzinotas is currently Full Professor / Chief Scientist I and Co-Head of the SIGCOM Research Group at SnT, University of Luxembourg. In the past, he has been a Visiting Professor at the University of Parma, Italy and he was involved in numerous Research and Development projects for the National Center for Scientific Research Demokritos, the Center of Research and Technology Hellas and the Center of Communication Systems Research, University of Surrey. He received the M.Eng. degree in telecommunications from the Aristotle University of Thessaloniki, Thessaloniki, Greece, in 2003, and the M.Sc. and Ph.D. degrees in electronic engineering from the University of Surrey, Surrey, U.K., in 2006 and 2009, respectively. He was a co-recipient of the 2014 IEEE Distinguished Contributions to Satellite Communications Award, the CROWNCOM 2015 Best Paper Award and the 2018 EURASIC JWCN Best Paper Award. He has (co-)authored more than 400 technical papers in refereed international journals, conferences and scientific books.

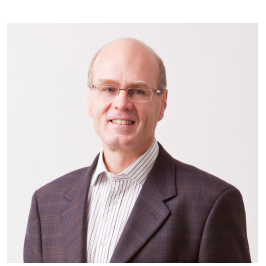

Björn Ottersten (S`87-M`89-SM`99-F`04) was born in Stockholm, Sweden, in 1961. He received the M.S. degree in electrical engineering and applied physics from Linkping University, Linkping, Sweden, in 1986, and the Ph.D. degree in electrical engineering from Stanford University, Stanford, CA, USA, in 1990. He has held research positions with the Department of Electrical Engineering, Linkping University, the Information Systems Laboratory, Stanford University, the Katholieke Universiteit Leuven, Leuven, Belgium, and the University of Luxembourg, Luxembourg. From 1996 to 1997, he was the Director of Research with ArrayComm, Inc., a start-up in San Jose, CA, USA, based on his patented technology. In 1991, he was appointed a Professor of signal processing with the Royal Institute of Technology (KTH), Stockholm, Sweden. From 1992 to 2004, he was the Head of the Department for Signals, Sensors, and Systems, KTH, and from 2004 to 2008, he was the Dean of the School of Electrical Engineering, KTH. He is currently the Director for the Interdisciplinary Centre for Security, Reliability and Trust, University of Luxembourg. He was a recipient of the IEEE Signal Processing Society Technical Achievement Award in 2011 and the European Research Council advanced research grant twice, in 20092013 and in 20172022. He has coauthored journal papers that received the IEEE Signal Processing Society Best Paper Award in 1993, 2001, 2006, and 2013, and seven IEEE conference papers best paper awards. He has served as an Associate Editor for the IEEE TRANSACTIONS ON SIGNAL PROCESSING and the Editorial Board of the IEEE Signal Processing Magazine. He is currently a member of the editorial boards of EURASIP Signal Processing Journal, EURASIP Journal of Advances Signal Processing and Foundations and Trends of Signal Processing. $\mathrm{He}$ is a fellow of EURASIP 